\title{
Astrochemistry of sub-millimeter sources in Orion ${ }^{\star}$
}

\section{Studying the variations of molecular tracers with changing physical conditions}

\author{
D. Johnstone ${ }^{1,2}$, A. M. S. Boonman ${ }^{3}$, and E. F. van Dishoeck ${ }^{3}$ \\ 1 National Research Council Canada, Herzberg Institute of Astrophysics, 5071 West Saanich Road, Victoria, B.C., \\ V9E 2E7, Canada \\ 2 Department of Physics \& Astronomy, University of Victoria, Victoria, BC, V8P 1A1, Canada \\ ${ }^{3}$ Leiden Observatory, PO Box 9513, 2300 RA Leiden, The Netherlands
}

Received 28 January 2003 / Accepted 29 August 2003

\begin{abstract}
Cornerstone molecules ( $\mathrm{CO}, \mathrm{H}_{2} \mathrm{CO}, \mathrm{CH}_{3} \mathrm{OH}, \mathrm{HCN}, \mathrm{HNC}, \mathrm{CN}, \mathrm{CS}, \mathrm{SO}$ ) were observed toward seven sub-millimeter bright sources in the Orion molecular cloud in order to quantify the range of conditions for which individual molecular line tracers provide physical and chemical information. Five of the sources observed were protostellar, ranging in energetics from 1-500 $L_{\odot}$, while the other two sources were located at a shock front and within a photodissociation region (PDR).

Statistical equilibrium calculations were used to deduce from the measured line strengths the physical conditions within each source and the abundance of each molecule. In all cases except the shock and the PDR, the abundance of CO with respect to $\mathrm{H}_{2}$ appears significantly below (factor of ten) the general molecular cloud value of $10^{-4}$. Formaldehyde measurements were used to estimate a mean temperature and density for the gas in each source. Evidence was found for trends between the derived abundance of $\mathrm{CO}, \mathrm{H}_{2} \mathrm{CO}, \mathrm{CH}_{3} \mathrm{OH}$, and $\mathrm{CS}$ and the energetics of the source, with hotter sources having higher abundances. Determining whether this is due to a linear progression of abundance with temperature or sharp jumps at particular temperatures will require more detailed modeling. The observed methanol transitions require high temperatures $(T>50 \mathrm{~K})$, and thus energetic sources, within all but one of the observed protostellar sources. The same conclusion is obtained from observations of the CS 7-6 transition. Analysis of the HCN and HNC 4-3 transitions provides further support for high densities $n>10^{7} \mathrm{~cm}^{-3}$ in all the protostellar sources.

The shape of the CO 3-2 line profile provides evidence for internal energetic events (outflows) in all but one of the protostellar sources, and shows an extreme kinematic signature in the shock region. In general, the CO line and its isotopes do not significantly contaminate the $850 \mu \mathrm{m}$ broadband flux (less than 10\%); however, in the shock region the CO lines alone account for more than two thirds of the measured sub-millimeter flux. In the energetic sources, the combined flux from all other measured molecular lines provides up to an additional few percent of line contamination.
\end{abstract}

Key words. ISM: individual objects: Orion A, Orion B - ISM: molecules - stars: formation

\section{Introduction}

The study of structure within the star-forming regions of molecular clouds has benefitted significantly from observations of many molecular lines, each tracing specific chemical and physical conditions. Most studies either focus on surveys using particular tracers, such as carbon monoxide, $\mathrm{N}_{2} \mathrm{H}^{+}$, or ammonia, in order to map the column density and kinematics of the gas (Myers 1999), or have focused on individual sources, producing detailed molecular catalogues in several to dozens of

Send offprint requests to: D. Johnstone, e-mail: doug.johnstone@nrc-cnrc.gc.ca

$\star$ Table 3 is only available in electronic form at http://www.edpsciences.org species designed to constrain the physical and chemical morphology of the region (van Dishoeck \& Blake 1998).

Several molecules have emerged as particularly good diagnostics of the conditions and chemistry near young stellar objects. For example, $\mathrm{H}_{2} \mathrm{CO}$ has many lines that are readily observed at sub-millimeter wavelengths, and whose ratios are either good temperature or density tracers (e.g., Hurt et al. 1996; Mangum et al. 1999; Mitchell et al. 2001). Analysis of dust continuum and line emission has shown that temperature and density gradients exist across the protostellar envelopes, with temperatures varying from the inner to the outer region from $>100 \mathrm{~K}$ to less than $20 \mathrm{~K}$ and densities from $>10^{7} \mathrm{~cm}^{-3}$ to $\sim 10^{4} \mathrm{~cm}^{-3}$ (e.g., van der Tak et al. 2000a; Shirley et al. 2002; Jørgensen et al. 2002). The chemistry responds to these 
changes. Molecules freeze-out onto the grains in the cold outer parts of the clouds, where they can form new species through grain-surface reactions. The composition of icy mantles can be determined through infrared absorption, with species such as $\mathrm{H}_{2} \mathrm{O}, \mathrm{CO}, \mathrm{CO}_{2}$ and $\mathrm{CH}_{3} \mathrm{OH}$ known to have high ice abundances (e.g., Gibb et al. 2000). In the inner region close to the protostar, the grains are heated and the ices are observed to evaporate back into the gas (e.g., van Dishoeck \& Helmich 1996; Boonman \& van Dishoeck 2003). High temperature gasphase reactions between evaporated species can subsequently lead to high abundances of complex organic molecules observed in high-mass hot cores (e.g., Rodgers \& Charnley 2001). Depending on the evolutionary state of the source, different chemical characteristics become more prominent.

In this paper we consider a selection of cornerstone molecules and study a variety of independent locations within the Integral Shaped Filament (ISF) in Orion A, along with NGC 2071IR in Orion B, in order to quantify the range of conditions for which individual molecular line tracers provide physical or chemical information. This is primarily a morphological study, comparing the differences in molecular line emission across sources of varying physical conditions using simplified equilibrium modeling, in order to search for obvious diagnostic features within the data. The list of sources observed in Orion A was compiled from a sensitive dust continuum survey of the ISF (Johnstone \& Bally 1999) and includes both highly enshrouded sub-millimeter bright sources, possibly protostellar, through more evolved protostars, a bright PDR knot, and a shock front. Many of the young stellar objects are of intermediate mass and thus the observations bracket sub-millimeter studies of high-mass (e.g. Hatchell et al. 1998; van der Tak et al. 2000a) and low-mass (e.g. Blake et al. 1994, 1995; van Dishoeck et al. 1995; Buckle \& Fuller 2002; Jørgensen et al. 2002) star-forming regions. The selection of cornerstone molecules and molecular transitions was chosen based on previous experience in detailed studies of individual objects.

\section{The Orion integral shaped filament}

The ISF (Bally et al. 1987) in the northern portion of the Orion A molecular cloud is the nearest site with active high-mass through low-mass star formation. The region is associated with the Orion Nebula and the Trapezium Cluster of 700 young stars (Hillenbrand 1997; Hillenbrand \& Hartmann 1998), contains the OMC1 cloud core immediately behind the Nebula, and two other extensively studied star-forming cores, OMC2 and OMC3 located about $15^{\prime}$ and $25^{\prime}$ to the north (Castets \& Langer 1994). To the south the region becomes much quieter with OMC4 (Johnstone \& Bally 1999) showing no bright sub-millimeter sources. The ISF has spawned several thousand young stars in the past few million years and contains dozens (possibly hundreds) of embedded young stellar objects (Chini et al. 1997; Johnstone \& Bally 1999) which power dozens of molecular outflows, Herbig-Haro objects (Reipurth et al. 1997), and molecular hydrogen emitting shocks and jets (Yu et al. 1997). The abundance of star formation, at various stages of evolution, within a single, nearby, molecular cloud

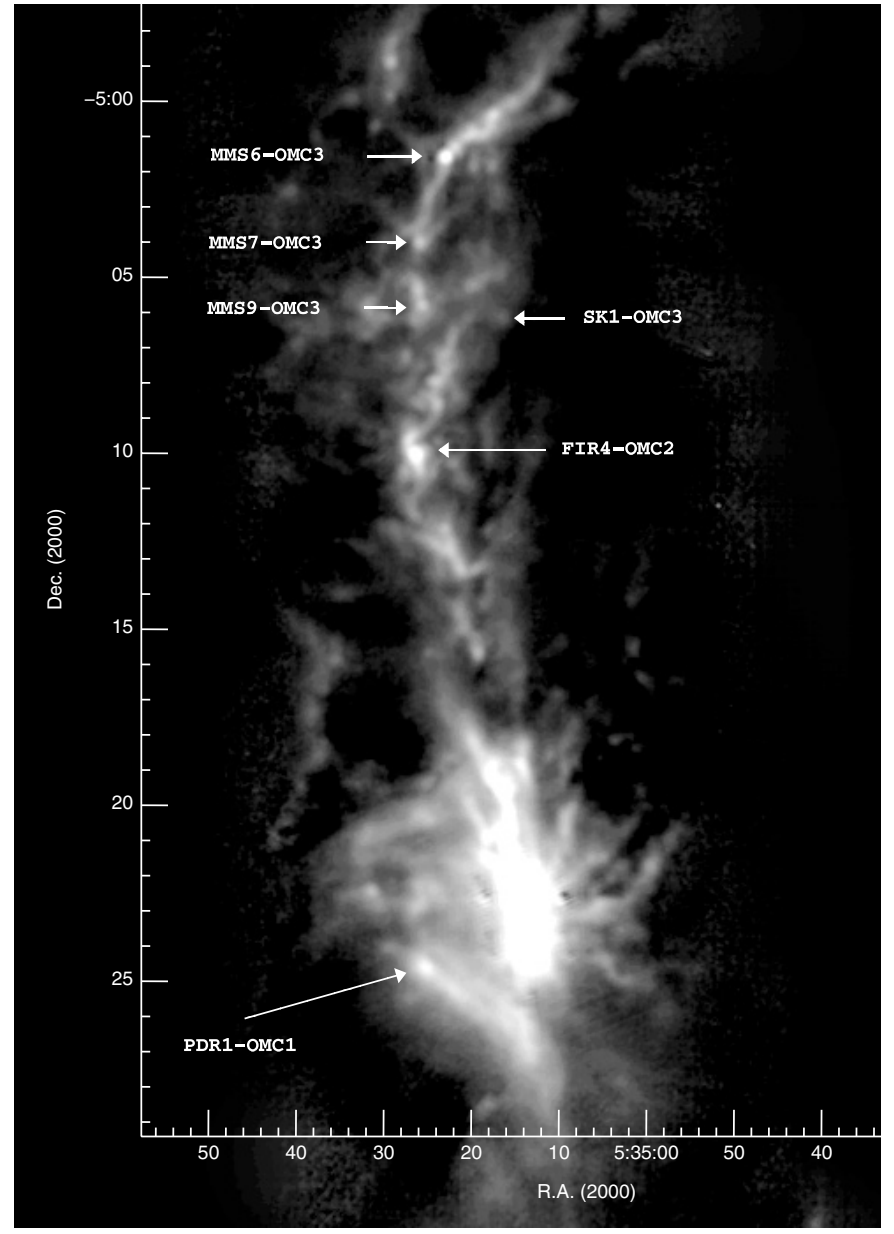

Fig. 1. The Integral Shaped Filament (ISF) at the north end of the Orion A molecular cloud, observed in dust continuum emission at 850 microns. The grey-scale ranges from 0 to 5 Jys with a logarithmic stretch. The positions of the six sources located in the ISF are indicated by arrows pointing at the peak continuum flux locations. Details of the sources are found in Table 1.

provides a unique setting for conducting tests of astrochemical diagnostics.

\subsection{The individual sources}

The sources chosen for this study were primarily drawn from the 450 and $850 \mu \mathrm{m}$ dust emission maps of the ISF (Johnstone \& Bally 1999; Fig. 1). Supplemental information on the properties of the sources comes from measurements at $350 \mu \mathrm{m}$ (Lis et al. 1998), $1300 \mu \mathrm{m}$ (Chini et al. 1997), $3.6 \mathrm{~cm}$ VLA data (Reipurth et al. 1999), and the IRAS point source catalogue. Additional $2 \mu \mathrm{m}$ molecular hydrogen observations (Yu et al. 1997) of the ISF provide evidence of local heating expected within shocks, and delineate the location of molecular jets. The seven sources were chosen to cover a wide selection of physical conditions in order to test the hypothesis that molecular line observations provide sensitive diagnostics.

The coldest sub-millimeter source in our sample is MMS6, $T_{\mathrm{d}} \sim 15 \mathrm{~K}$ as measured by the sub-millimeter fluxes from 350 to $1300 \mu \mathrm{m}$ (Lis et al. 2001). MMS6 is also the brightest $850 \mu \mathrm{m}$ source within the OMC3 region of the ISF, with 
a peak flux of $7.4 \mathrm{Jy}$ within a 15 arcsec beam (Johnstone \& Bally 1999). The total mass within the envelope is estimated to be $36 M_{\odot}$ (Chini et al. 1997). There is no corresponding IRAS point source, although the background brightness of the region makes point source detection difficult, suggesting that there is no strong internal heating source. The sub-millimeter luminosity, energy emitted at wavelengths greater than $350 \mu \mathrm{m}$, is $1.2 L_{\odot}$; however, the lack of far-infrared emission measurements provides only an upper limit on the total bolometric luminosity of $<60 L_{\odot}$ (Chini et al. 1997). Despite the very cold appearance of MMS6 in the sub-millimeter, and thus the possibility that it is a pre-stellar clump heated only from the outside by the strong interstellar radiation field (ISRF) in Orion, MMS6 has been classified as a Class 0 protostar (Chini et al. 1997). Yu et al. (1997) observed knots of $\mathrm{H}_{2}$ directly north and south of MMS6 which are interpreted as bow shocks within a molecular outflow from the continuum source. Chini et al. (1997) report substantial high-velocity red-shifted emission in ${ }^{12} \mathrm{CO}$ centered on MMS6, although no corresponding blue-shifted lobe was observed. More recent observations by Aso et al. (2000) associate the $\mathrm{CO}$ outflow with a different sub-millimeter source. VLA observations at $3.6 \mathrm{~cm}$ (Reipurth et al. 1999) locate a radio source coincident with MMS6. Such coincidences are common among Class 0 sources and have been interpreted as thermal Bremsstrahlung radiation produced in shocks within the inner part of the molecular outflow. Thus, while MMS6 is possibly a pre-collapse clump, it is likely that the sub-millimeter source enshrouds a very young Class 0 protostar.

Despite being three times fainter than MMS6 at $850 \mu \mathrm{m}$, MMS9 appears to be the source of the most powerful outflow in the OMC2/3 region (Yu et al. 2000). The spectral index for MMS9 is less steep than observed in MMS6, suggesting a warmer temperature in the dust envelope, $T_{\mathrm{d}} \sim 20 \mathrm{~K}$ (Chini et al. 1997). The total envelope mass is estimated to be $10 M_{\odot}$, considerably lower than the envelope mass of MMS6. The IRAS flux limits constrain the luminosity of the embedded source to be $<94 L_{\odot}$ (Chini et al. 1997). Despite having no coincident IRAS point source, MMS9 is expected to contain a well-advanced Class 0 protostar. Reipurth et al. (1999) observed $3.6 \mathrm{~cm}$ continuum emission at this location. The rising infrared background toward MMS9 may be responsible for the lack of an IRAS point source detection.

The only IRAS point source within the OMC3 region is IRAS 05329-0505, coincident with MMS7. From the wavelength of the peak emission, $\lambda_{\max } \sim 60 \mu \mathrm{m}$, the warm component of the dust can be estimated, $T_{\mathrm{d}} \sim 50 \mathrm{~K}$. In the submillimeter MMS7 appears quite similar to MMS9, although the envelope temperature is somewhat higher $T_{\mathrm{d}} \sim 26 \mathrm{~K}$ (Chini et al. 1997). The total envelope mass is $\sim 8 M_{\odot}$, comparable to the envelope around MMS9, and the bolometric luminosity is $\sim 76 L_{\odot}$. Reipurth et al. (1999) observed $3.6 \mathrm{~cm}$ continuum emission at this location, providing further evidence for an embedded Class 0 source. MMS7 appears to be in a somewhat more advanced stage of star formation, powering a giant bipolar Herbig-Haro flow (Reipurth et al. 1997) and harboring both a central FU Ori-like source (Reipurth \& Aspin 1997) and an optical reflection nebula (Wolstencroft et al. 1986).
Further south along the ridge, near the center of the OMC2 molecular core, lies the far infrared source FIR4 (Mezger et al. 1990), also observed as an IRAS point source. The region around FIR4 harbors a cluster of near-infrared sources (Johnson et al. 1990) although none are explicitly coincident with the far-infrared source. FIR4 is the brightest submillimeter source in the OMC2 region and contains a VLA radio source (Reipurth et al. 1999), leading to identification as a Class 0 object. Its proximity to the near IR cluster suggests, however, that it might be a more evolved envelope. The presence of far-infrared emission provides evidence for warm dust $T_{\mathrm{d}} \sim 40 \mathrm{~K}$ (Mezger et al. 1990), although the sub-millimeter emission traces the much colder outer envelope $T_{\mathrm{d}} \sim 15 \mathrm{~K}$. The total envelope mass is $\sim 34 M_{\odot}$ and the luminosity is $\sim 400 L_{\odot}$ (Mezger et al. 1990).

The final protostellar source in our sample is NGC 2071IR in the Orion B North molecular cloud (Johnstone et al. 2001; Motte et al. 2001; Harvey et al. 1979). Significant far-infrared emission from the source (Butner et al. 1990) provides evidence of an internal source and a warm dust $T_{\mathrm{d}}>50 \mathrm{~K}$ component providing a reprocessed luminosity $\sim 500 L_{\odot}$. The total envelope mass, derived from the sub-millimeter dust emission, is $12-30 M_{\odot}$ (Johnstone et al. 2001). NGC 2071 contains one of the most powerful outflows known (Bally \& Lada 1983).

Along with the five protostellar candidates, two submillimeter bright regions which are not associated with individual protostars were also observed. A sub-millimeter bright location, PDR1, along the warm photodissociation region (the Orion Bar) separating the HII region M42 (the Orion Nebula) from the Orion A molecular cloud was included to monitor changes in the molecular line diagnostics in regions of enhanced UV fluxes and higher excitation temperatures (see also Hogerheijde et al. 1995). Also, a strong shock front, SK1, located via bright $2 \mu \mathrm{m} \mathrm{H}_{2}$ emission (Yu et al. 1997), was included to test for shock diagnostics. The shocked region is also visible in the spectral index map of the ISF (Johnstone \& Bally 1999 ) as a region with anomalously shallow slope, requiring either a lower temperature than the surrounding material, a shallower dust emissivity profile, or contamination of the $850 \mu \mathrm{m}$ continuum measurement by molecular lines.

A summary of the physical properties of the seven sources is presented in Table 1. The sources are ordered from the coldest, least energetic, to the warmest, most energetic, with the PDR and shock front noted separately.

\section{Observations}

\subsection{Choice of molecules}

Eight different molecules and their isotopes have been observed in order to find useful diagnostics to study physical and chemical differences between the sources in our sample. Among these are carbon-, oxygen-, nitrogen-, and sulphurbearing species, allowing for a study of differentiation in the chemical abundances. Abundant $\mathrm{CO}$ is observed toward all sources. Nevertheless, it is a useful molecule to study depletion effects, which are expected to be largest for the coldest, less evolved sources. In those sources where CO is not too depleted, it quickly becomes optically thick. In that case, 
Table 1. Source list and sub-millimeter diagnostics.

\begin{tabular}{lcccccc}
\hline \hline Name & $\begin{array}{c}\mathrm{RA}^{a} \\
(\mathrm{~J} 2000)\end{array}$ & $\begin{array}{c}\operatorname{Dec}^{a} \\
(\mathrm{~J} 2000)\end{array}$ & $\begin{array}{c}T_{\mathrm{d}} \\
(K)\end{array}$ & $\begin{array}{c}M_{\mathrm{env}} \\
\left(M_{\odot}\right)\end{array}$ & $\begin{array}{c}L \\
\left(L_{\odot}\right)\end{array}$ & Object \\
\hline MMS6-OMC3 & $5: 35: 23.5$ & $-05: 01: 32$ & 15 & 36 & $1.2-60$ & Class 0 \\
MMS9-OMC3 & $5: 35: 26.2$ & $-05: 05: 46$ & 20 & 10 & $0.6-90$ & Class 0 \\
MMS7-OMC3 & $5: 35: 26.4$ & $-05: 03: 59$ & $26-50$ & 8 & 76 & Class 0 \\
FIR4-OMC2 & $5: 35: 26.7$ & $-05: 09: 59$ & $15-40$ & 34 & 400 & Class 0/I \\
NGC 2071IR & $5: 47: 04.4$ & $+00: 21: 49$ & $20-50$ & 30 & 500 & Class 0/I \\
PDR1-OMC1 & $5: 35: 25.3$ & $-05: 24: 34$ & 60 & & & PDR \\
SK1-OMC3 & $5: 35: 17.0$ & $-05: 06: 03$ & 10 & & & Shock \\
\hline
\end{tabular}

${ }^{a}$ Positions are taken from the sub-millimter maps of the ISF (Johnstone \& Bally 1999) and Orion B North (Johnstone et al. 2001).

Table 2. Calendar of observations and conditions.

\begin{tabular}{|c|c|c|c|c|c|c|}
\hline Date & Receiver & $\begin{array}{c}v_{\text {upper }} \\
(\mathrm{GHz})\end{array}$ & $\begin{array}{c}v_{\text {lower }} \\
(\mathrm{GHz})\end{array}$ & $\begin{array}{c}\Delta V \\
\left(\mathrm{~km} \mathrm{~s}^{-1}\right)\end{array}$ & $\begin{array}{l}T_{\text {sys }} \\
(\mathrm{K})\end{array}$ & Lines \\
\hline 1999 January & RxA3 & 218.35 & 226.48 & 0.2 & $400-700$ & $\mathrm{H}_{2} \mathrm{CO}, \mathrm{CH}_{3} \mathrm{OH}$ \\
\hline 1999 September & $\mathrm{RxB} 3$ & 330.53 & 338.53 & 0.5 & $1200-1700$ & ${ }^{13} \mathrm{CO}, \mathrm{CH}_{3} \mathrm{OH}$ \\
\hline 1999 September & RxB3 & 338.53 & 346.53 & 0.5 & $1200-1700$ & $\mathrm{CH}_{3} \mathrm{OH}, \mathrm{SO}$ \\
\hline 1999 September & RxB3 & 354.40 & 362.40 & 0.5 & $1200-1700$ & $\mathrm{HCN}, \mathrm{HNC}$ \\
\hline 2000 December & $\mathrm{RxA} 3$ & 233.00 & 241.00 & 0.4 & $350-400$ & $\mathrm{C}^{34} \mathrm{~S}$ \\
\hline 2000 December & $\mathrm{RxA} 3$ & 233.75 & 241.75 & 0.4 & $350-400$ & $\mathrm{CH}_{3} \mathrm{OH}$ \\
\hline 2000 December & $\mathrm{RxA} 3$ & 236.94 & 244.94 & 0.4 & $375-385$ & CS \\
\hline 2000 December & $\mathrm{RxB} 3$ & 337.25 & 345.25 & 0.5 & $530-550,710-740$ & $\mathrm{C}^{17} \mathrm{O}, \mathrm{C}^{34} \mathrm{~S}$ \\
\hline 2000 December & $\mathrm{RxB} 3$ & 340.45 & 348.45 & 0.5 & $700-800$ & $\mathrm{CN}, \mathrm{HN}^{13} \mathrm{C}$ \\
\hline 2000 December & $\mathrm{RxB} 3$ & 345.67 & 353.67 & 0.5 & $490-510$ & $\mathrm{CO}$ \\
\hline 2000 December & $\mathrm{RxB} 3$ & 354.60 & 362.60 & 0.5 & $590-620$ & $\mathrm{HCN}, \mathrm{HNC}, \mathrm{H}_{2} \mathrm{CO}$ \\
\hline
\end{tabular}

the optically thin isotope $\mathrm{C}^{17} \mathrm{O}$ can be used to derive $\mathrm{H}_{2}$ column densities.

Formaldehyde, $\mathrm{H}_{2} \mathrm{CO}$, is a slightly asymmetric rotor molecule, providing it with many of the temperature and density diagnostic features of purely symmetric rotors. Mangum \& Wootten (1993) and van Dishoeck et al. (1993) list many of the strengths of this molecule as a probe of the physical conditions in dense molecular clouds. Methanol, $\mathrm{CH}_{3} \mathrm{OH}$, is a complex molecule with many transitions in the sub-millimeter. Since these lines are concentrated in complex bands spanning relatively small wavelength ranges, this allows for efficient observations even at relatively high spectral resolution. Methanol is also chemically important, since it has been detected both in the gas-phase and on grains (e.g. Dartois et al. 1999). The high gas-phase abundances of $\mathrm{CH}_{3} \mathrm{OH}$ most likely result from evaporation off of grains (Charnley et al. 1992; van der Tak et al. 2000b; Schöier et al. 2002).

One of the most abundant nitrogen-bearing species is HCN. Chemical models predict that the $\mathrm{HCN}$ abundances increase with temperature (Rodgers \& Charnley 2001), thus providing a useful evolutionary indicator. Observations of massive star-forming regions confirm this (Lahuis \& van Dishoeck 2000), although the situation for low-mass sources is less clear (Jørgensen et al., in prep.). Schilke et al. (1992) show that the abundance ratio of HCN and HNC depends on the density and kinetic temperature of the gas, providing a physical diagnostic.
The chemical equilibrium of $\mathrm{HCN}, \mathrm{HNC}$, and $\mathrm{CN}$ also depends on the hardness of the radiation field.

Finally, sulphur-bearing species have been associated with shocks and/or outflows (e.g. Orion; Blake et al. 1987). However, recent studies of sulphur-bearing species in massive star-forming regions other than Orion cannot distinguish between an origin in the circumstellar envelope or the outflow (Keane et al. 2001a; van der Tak et al. 2003). Thus, it is still debatable whether sulphur-bearing species are good diagnostics of outflows. On the other hand, CS transitions have been used to derive detailed density structure within the molecular envelopes of star-forming regions, making this species a useful indicator of physical properties (van der Tak et al. 2000a). The $\mathrm{H}_{2} \mathrm{~S} / \mathrm{SO}_{2}$ ratio has also been proposed as a chemical clock of hot core regions (Charnley 1997), although observational results are still ambiguous (Hatchell et al. 1998; van der Tak et al. 2003).

\subsection{Sub-millimeter observations}

The molecular line observations were taken at the 15-meter James Clerk Maxwell telescope $\left(\mathrm{JCMT}^{1}\right.$ on Mauna Kea in

1 The JCMT is operated by the Joint Astronomy Centre on behalf of the Particle Physics and Astronomy Research Council of the UK, The Netherlands Organization for Scientific Research, and the National Research Council of Canada. 

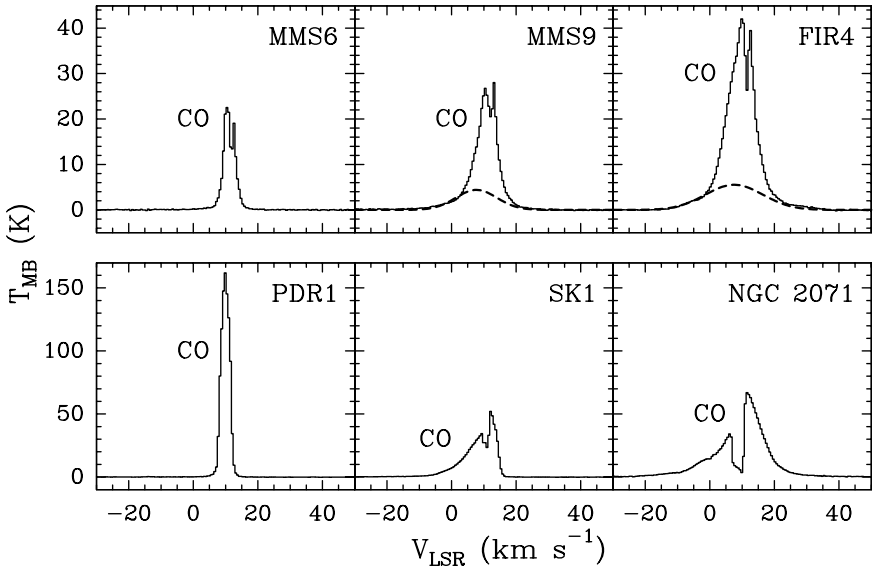

Fig. 2. The $\mathrm{CO} J=3-2$ line profiles. Note the self-absorption in all sources except PDR1, and the extended line wings (dashed lines) in all sources except MMS6, and PDR1.

Hawaii. The data were obtained during three separate observing runs: January 1999, September 1999, and December 2000. Table 2 provides details of the instruments and settings used during each run, along with typical receiver conditions. For each source, Table 3 lists the molecular lines observed and the results of fitting one or two Gaussian components to the integrated line intensity, the full width at half maximum, and the peak intensity.

The data were obtained in a beam switching mode, with a 10 arcmin switch in right ascension. The integrated line data were transfered from the antenna temperature scale $T_{\mathrm{A}}^{*}$ to the main-beam brightness scale $T_{\mathrm{mb}}$ by dividing by the main-beam efficiency $\eta_{\mathrm{mb}}$. For the $A$ band receiver $(210-270 \mathrm{GHz})$ the measured efficiency is $\eta_{\mathrm{mb}}=0.69$, while for the $B$ band receiver $(330-370 \mathrm{GHz}) \eta_{\mathrm{mb}}=0.60$. The relevant beam sizes for the JCMT are 21 and 14 arcsec at respectively 230 and $345 \mathrm{GHz}$. The velocity resolution ranged from $0.2-0.5 \mathrm{~km} \mathrm{~s}^{-1}$. The rms noise after a typical integration time of twenty minutes was $0.30 \mathrm{~K}$ per channel for $A$ band observations and $0.15 \mathrm{~K}$ per channel for $B$ band observations. Further details on the JCMT and the heterodyne receivers can be found on the JCMT homepage $^{2}$.

\subsection{Representative line profiles}

Many important differences between the sources are readily observed simply by looking at the spectra. The extremely strong, and almost always self-absorbed, $\mathrm{CO} 3-2$ transition reveals information on the gas kinematics within each source (Fig. 2). Single component Gaussian fits to the profiles are only possible for MMS6 (line width $\left.4 \mathrm{~km} \mathrm{~s}^{-1}\right)$ and PDR1 $\left(3 \mathrm{~km} \mathrm{~s}^{-1}\right)$. MMS9 requires both a narrow component $\left(6 \mathrm{~km} \mathrm{~s}^{-1}\right)$, providing two thirds of the integrated intensity, and a broad component $\left(15 \mathrm{~km} \mathrm{~s}^{-1}\right)$. FIR4 appears to be a scaled-up version of MMS9, with a narrow component $\left(9 \mathrm{~km} \mathrm{~s}^{-1}\right)$ containing three quarters of the integrated intensity and a broad component $\left(21 \mathrm{~km} \mathrm{~s}^{-1}\right)$. Both the shock region and NGC 2071 are poorly fit by Gaussian components and are dominated by the extended wings.

\footnotetext{
${ }^{2}$ http://www.jach.hawaii.edu/JACpublic/JCMT
}

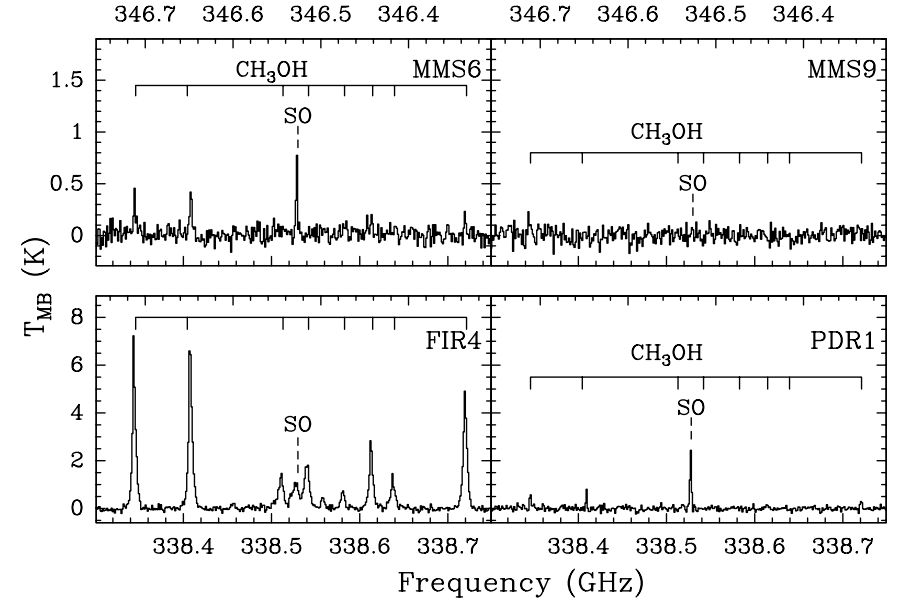

Fig. 3. A selection of methanol lines observable with a single spectral setting, along with an $\mathrm{SO} N_{J}=8_{9} \rightarrow 7_{8}$ line.

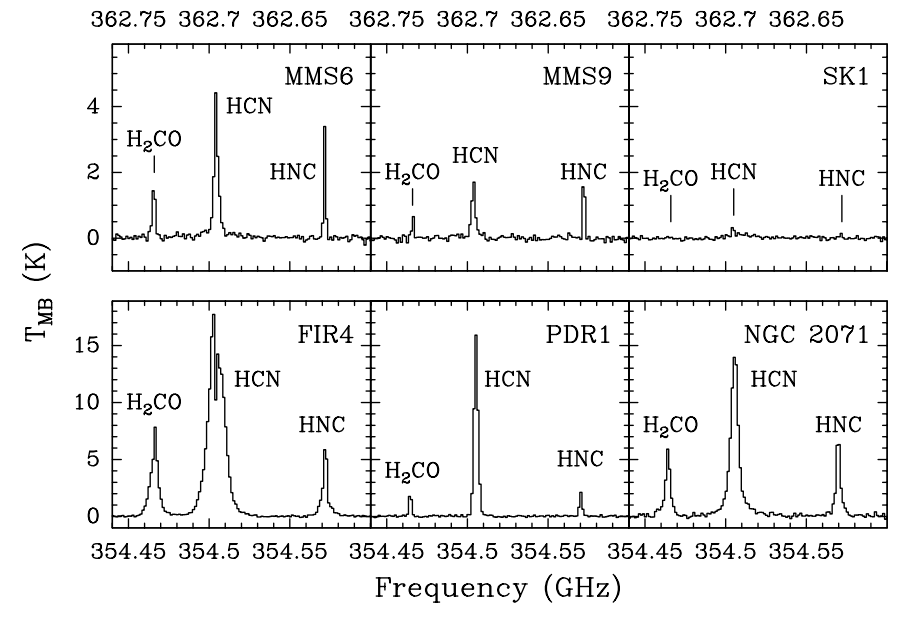

Fig. 4. The $\mathrm{HCN}$ and $\mathrm{HNC} J=4-3$ transitions.

Eight of the methanol lines are observed in a single spectral setting and can be plotted for each source (see examples in Fig. 3). These spectra also contain the sole observed SO transition. In agreement with the results found by van der Tak et al. (2003) there is an apparent lack of correlation between the strength of the SO line and the methanol lines. As well, differences in the relative strengths of the methanol lines are observable between MMS6 and FIR4, indicating the more extreme temperature and density conditions in the energetic infrared source. Finally, the FIR4 methanol spectra show evidence for two components, narrow and broad, similar to the $\mathrm{CO}$ spectra.

The HCN and HNC 4-3 profiles are also observed in a single spectral setting (Fig. 4). A range of relative line strengths is seen in the spectra; however, optical depth considerations make simple abundance determinations more complicated (see Sect. 4.5.1). The substantial optical depth of the transitions allows for more detailed determinations of the source kinematics. In all sources except PDR1, the HCN line profile is broader than the HNC line profile or has evidence for extended line wings. 


\section{Molecular line survey}

\subsection{Statistical equilibrium calculations}

The analysis of the data is done with statistical equilibrium calculations, using the numerical code RADEX $^{3}$. The adopted method is described in detail by Jansen et al. (1994), van Dishoeck et al. (1993), and Jansen et al. (1995). This method assumes that the source is a homogeneous spherical region. The decoupling of radiative transfer and level populations is done with the escape probability method. The optical depth $\tau$ at the line center is proportional to the ratio of the total column density and the line width of the molecule. The optical depth and the line intensity depend only on this ratio. All variables can be calculated, using a geometrical assumption that they are constant throughout the region. The radiative excitation of rotational lines in the sub-millimeter region is usually dominated by the $2.73 \mathrm{~K}$ cosmic background radiation, which peaks at $1.871 \mathrm{~mm}$. Therefore, this external radiation field is included in the calculations. No central radiation field is included nor infrared pumping by dust. Since the sources have rather low dust temperatures, this latter effect is expected to be negligible.

The kinetic temperature $T_{\text {kin }}$ and the density $n\left(\mathrm{H}_{2}\right)$ for the homogeneous region can be estimated from line ratio calculations where available. The column density of molecule $X$, $N(X)$, can then be estimated using the temperature and the density as found from line ratio calculations, such that the line width, integrated line strength, and main beam temperature of the emission lines can be fitted.

Due to the simplistic treatment of the envelope structure the derived abundances are only roughly determined. Rising temperature and density gradients toward the envelope centers are expected due to heating by the central protostellar sources and these conditions can influence the observed line strengths significantly. Additionally, these Orion sources reside within an enhanced external radiation field, due to the proximity of many nearby massive stars (Li et al. 2003). Thus, the outer envelopes are kept warm, $T_{\mathrm{d}} \sim 30 \mathrm{~K}$ (Johnstone \& Bally 1999). Modeling of such envelopes ( $\mathrm{Li}$ et al. 2003; Jørgensen et al., in prep.) reveal an inward decreasing temperature gradient near the surface, encompassing much of the mass of the envelope, coupled to an inward rising temperature gradient once the central heating source dominates.

The large, externally warmed, envelopes surrounding the protostellar sources in Orion should allow for reasonable abundance estimates from the observed line intensities using statistical equilibrium calculations, except for those transitions requiring either very high densities $n>10^{7} \mathrm{~cm}^{-3}$ or high temperatures $T>50 \mathrm{~K}$. As a guide to the accuracy of the calculations presented in this paper, detailed modeling of the protostellar source IRAS 16293-2422, including careful derivation of the physical structure, has shown that abundance estimates from this simpler procedure are typically only accurate to within a factor of two or three (Schöier et al. 2002).

\footnotetext{
3 This program was originally written by J. H. Black. Here a more recent version by D. J. Jansen is used.
}

\subsection{Isotopes of carbon monoxide}

Within star-forming regions of molecular clouds, comparison between the line strengths of low-lying CO transitions and the intensity of the sub-millimeter dust continuum often suggest that there is a depletion of $\mathrm{CO}$ with respect to the dust, and by extension the molecular hydrogen abundance (see for example Mitchell et al. 2001; Jørgensen et al. 2002; Bergin et al. 2002; Bacmann et al. 2002). The most common explanation for this observational discrepancy between $\mathrm{CO}$ line strength and dust continuum emission is that the $\mathrm{CO}$ has depleted through freeze-out onto the grains. The time scale for depletion scales inversely with the density of the region, and is shorter than $10^{5} \mathrm{yr}$ for densities above a few $\times 10^{4} \mathrm{~cm}^{-3}$. Recent laboratory experiments on $\mathrm{CO}$ and $\mathrm{H}_{2} \mathrm{O}$ ices show that $\mathrm{CO}$ will remain completely frozen out if the grain temperatures stay below $\sim 20 \mathrm{~K}$. Above $20 \mathrm{~K}, \mathrm{CO}$ starts to evaporate back into the gas phase in a complicated pattern depending on the ice structure, with a fraction of the $\mathrm{CO}$ remaining trapped in the $\mathrm{H}_{2} \mathrm{O}$ ice matrix until the entire mantle evaporates at $\sim 100 \mathrm{~K}$ (Collings et al. 2003; Fraser et al. 2001).

\subsubsection{Line strengths}

Table 4 details the observed line strengths of $\mathrm{CO},{ }^{13} \mathrm{CO}$, and $\mathrm{C}^{17} \mathrm{O} 3-2$ transitions obtained at the JCMT, along with an estimate of the column of carbon monoxide along the line-ofsight. Using RADEX, the column is deduced from the integrated line strength of the $\mathrm{C}^{17} \mathrm{O} 3-2$ line, assuming that the ${ }^{13} \mathrm{CO}$ peak temperature provides a reasonable estimate of the kinetic temperature of the gas. The RADEX calculations for CO include energy levels up to $J=25$ and use collisional rate coefficients by Schinke et al. (1985). The results are not significantly changed if the new rate coefficients by Flower (2001) are adopted (see Jørgensen et al. 2002). For NGC 2071, where the ${ }^{13} \mathrm{CO}$ line was not observed, the temperature was assumed to be $30 \mathrm{~K}$, similar to the temperature of FIR4.

The cosmic abundance ratio for isotopes of oxygen, $\left[{ }^{16} \mathrm{O}\right] /\left[{ }^{17} \mathrm{O}\right] \sim 1800$ (Wilson \& Rood 1994), was used to convert the calculated $\mathrm{C}^{17} \mathrm{O}$ column to an equivalent column of $\mathrm{CO}$. For the expected conditions within the sub-millimeter clumps, $n>10^{4} \mathrm{~cm}^{-3}$, there is only a slight density dependence on the calculated column of the gas. In most cases the $\mathrm{CO}$ peak temperature was larger than the ${ }^{13} \mathrm{CO}$ temperature; however, the optical thickness of $\mathrm{CO}$ is extreme and hence the line profile may be dominated by a small fraction of hot gas associated with each source. There is still uncertainty comparing the thicker ${ }^{13} \mathrm{CO}$ with the thinner $\mathrm{C}^{17} \mathrm{O}$. We estimate the reliability of the computed physical parameters by comparing the RADEX computed optical depth of the ${ }^{13} \mathrm{CO}$ line, $\tau_{\text {mod }}$, with the observationally determined optical depth $\tau_{\text {obs }}$, measured by comparing the peak temperature of the $\mathrm{C}^{17} \mathrm{O}$ against the ${ }^{13} \mathrm{CO}$ peak temperature and assuming $\left[{ }^{13} \mathrm{CO}\right] /\left[\mathrm{C}^{17} \mathrm{O}\right]=25$ (Wilson $\&$ Rood 1994). This calculation confirms that the ${ }^{13} \mathrm{CO}$ line is optically thick in all cases $\left(\tau_{{ }^{13} \mathrm{CO}}>1.3\right)$. While there are deviations between the two determinations of the optical depth, the order unity dispersion provides an equivalent estimate of the reliability of the measured column density of $\mathrm{CO}$. 
Table 4. Physical conditions discerned from carbon-monoxide.

\begin{tabular}{|c|c|c|c|c|c|c|c|c|c|}
\hline \multirow[b]{2}{*}{ Source } & \multicolumn{2}{|l|}{$\mathrm{CO}$} & \multicolumn{2}{|l|}{${ }^{13} \mathrm{CO}$} & \multicolumn{2}{|l|}{$\mathrm{C}^{17} \mathrm{O}$} & \multirow[b]{2}{*}{$\begin{array}{c}N(\mathrm{CO}) \\
\left(10^{18} \mathrm{~cm}^{-2}\right)\end{array}$} & \multirow[b]{2}{*}{$\begin{array}{c}\tau_{\text {mod }} \\
{ }^{13} \mathrm{CO}\end{array}$} & \multirow[b]{2}{*}{$\tau_{\mathrm{obs}} / \tau_{\mathrm{mod}}$} \\
\hline & $\begin{array}{c}T_{\text {peak }} \\
(\mathrm{K})\end{array}$ & $\begin{array}{c}T \Delta V \\
\left(\mathrm{~K} \mathrm{~km} \mathrm{~s}^{-1}\right)\end{array}$ & $\begin{array}{c}T_{\text {peak }} \\
(\mathrm{K})\end{array}$ & $\begin{array}{c}T \Delta V \\
\left(\mathrm{~K} \mathrm{~km} \mathrm{~s}^{-1}\right)\end{array}$ & $\begin{array}{c}T_{\text {peak }} \\
(\mathrm{K})\end{array}$ & $\begin{array}{c}T \Delta V \\
\left(\mathrm{~K} \mathrm{~km} \mathrm{~s}^{-1}\right)\end{array}$ & & & \\
\hline MMS6 & 23. & 93. & 21. & 45. & 3.6 & 4.8 & 4.6 & 2.1 & 2.4 \\
\hline MMS9 & 28. & 225 & 23. & 59. & 2.1 & 3.0 & 2.7 & 1.3 & 1.5 \\
\hline FIR4 & 42. & 442 & 28. & 101 & 2.7 & 4.1 & 3.4 & 1.8 & 1.3 \\
\hline NGC 2071 & 67. & 770 & - & - & 2.2 & 7.3 & 5.7 & 1.5 & 1.0 \\
\hline PDR1 & 165 & 535 & 105 & 220 & 7.2 & 10. & 11. & 2.0 & 1.1 \\
\hline SK1 & 52. & 404 & 26. & 75. & 1.9 & 3.3 & 2.8 & 1.5 & 0.9 \\
\hline
\end{tabular}

Table 5. Measured total line intensity within the $850 \mu \mathrm{m}$ band and contribution to continuum flux.

\begin{tabular}{lccccc}
\hline \hline Source & $\begin{array}{c}\text { CO Line Strength } \\
\left(\mathrm{K} \mathrm{km} \mathrm{s}^{-1}\right)\end{array}$ & $\begin{array}{c}\text { Total Line Strength } \\
\left(\mathrm{K} \mathrm{km} \mathrm{s}^{-1}\right)\end{array}$ & $\begin{array}{c}\text { Contamination } \\
\%\end{array}$ & $\begin{array}{c}N\left(\mathrm{H}_{2}\right) \\
\left(10^{22} \mathrm{~cm}^{-2}\right)\end{array}$ & $\begin{array}{c}{[\mathrm{CO}] /\left[\mathrm{H}_{2}\right]} \\
\left(10^{-4}\right)\end{array}$ \\
\hline MMS6 & 143 & 170 & 1.5 & 104 & 0.04 \\
MMS9 & 287 & 301 & 8.2 & 14 & 0.19 \\
MMS7 & & & & 22 & \\
FIR4 & 547 & 934 & 8.0 & 47 & 0.07 \\
NGC 2071 & 777 & $945^{c}$ & 8.3 & 62 & 0.09 \\
& & & & & \\
PDR1 & 765 & 853 & 11.1 & 10 & 1.10 \\
SK1 & 483 & 487 & 63. & 6 & 0.47 \\
\hline
\end{tabular}

${ }^{a} \mathrm{CO}$ line strengths include contribution from $\mathrm{CO},{ }^{13} \mathrm{CO}$, and $\mathrm{C}^{17} \mathrm{O} J=3-2$ transitions.

${ }^{b}$ Total line strength includes all measured lines within the $850 \mu \mathrm{m}$ passband.

${ }^{c}$ The total line strength for NGC 2071 does not include contributions from methanol. Comparison with FIR4 suggests that an additional few percent of line contamination might be produced by these unobserved lines.

\subsubsection{Determination of $\mathrm{CO}$ abundance}

The column density of molecular hydrogen within each envelope is best determined from the sub-millimeter flux measurements. Assuming that the $850 \mu \mathrm{m}$ emission observed by Johnstone \& Bally (1999) and Johnstone et al. (2001) traces the bulk of the envelope and that the dust emission is optically thin, the mean column density through the center of each clump is related to the peak brightness:

$N\left(\mathrm{H}_{2}\right)=\frac{S_{850} \Omega_{\mathrm{bm}}}{B_{850}\left(T_{\mathrm{d}}\right) \kappa_{850} \mu m_{\mathrm{H}_{2}}}$,

where $\Omega_{\mathrm{bm}}$ is the effective solid angle of the SCUBA beam (in steradians), $B_{850}\left(T_{\mathrm{d}}\right)$ is the Planck function at $850 \mu \mathrm{m}$ for dust radiating at $T_{\mathrm{d}}, \kappa_{850}$ is the dust emissivity at $850 \mu \mathrm{m}$ (assumed here to be $\kappa_{850}=0.02 \mathrm{~cm}^{2} \mathrm{~g}^{-1}$ ), $m_{\mathrm{H}_{2}}$ is the molecular hydrogen mass, and $\mu$ is the mean molecular weight (in units of $m_{\mathrm{H}_{2}}$ ).

As shown in Appendix A, significant CO line flux can contaminate the broadband continuum measurements; therefore, it is important to correct the continuum measurements for the presence of line emission. Having both line and continuum measurements for each of the sources in Orion allows for a direct measure of the level of this line contamination. At $850 \mu \mathrm{m}, 1 \mathrm{Jy}$ within the JCMT $14 \mathrm{arcsec}$ beam is equivalent to a brightness temperature of $51 \mathrm{mK}$. However, the broad SCUBA $850 \mu \mathrm{m}$ bandpass, $30 \mathrm{GHz}$ wide, dilutes the importance of individual spectral line features against the continuum. The equivalent line flux required to mimic a $1 \mathrm{Jy}$ signal with SCUBA on the JCMT is $1540 \mathrm{~K} \mathrm{~km} \mathrm{~s}^{-1}$. For a typical peak gas temperature of $\sim 25 \mathrm{~K}$, the Gaussian component of the CO line would need to be $\sim 6 \mathrm{~km} \mathrm{~s}^{-1}$ wide to produce a ten percent, $0.1 \mathrm{Jy}$, error in the continuum measurement. Thus, for strong continuum sources, $S>2 \mathrm{Jy}$, line contamination should be minimal. Table 5 presents the $\mathrm{CO}$ line contamination for each of the six Orion sources. For completeness, the combined line contamination due to all other observed lines lying within the $850 \mu \mathrm{m}$ bandpass is also shown. With the exception of the shock knot SK1, the total line contamination is always less than about ten percent. Note that SK1 has a high peak brightness temperature for the $\mathrm{CO}$ 3-2 transition and has very extended line wings, consistent with its identification as a hot shock front by Yu et al. (1997) on the basis of bright $\mathrm{H}_{2}$ emission.

After accounting for line contamination, it is straightforward to compute the column density of $\mathrm{H}_{2}$, derived from the strength of the remaining continuum emission, and the fractional abundance of $\mathrm{CO}$ for each source. These values are also presented in Table 5. Within the PDR and the shock front, the abundance of gas-phase $\mathrm{CO}$ agrees with the expected value of $10^{-4}$ to within the accuracy of the computations (a factor of a few). However, toward each of the sub-millimeter protostar regions the $\mathrm{CO}$ gas-phase abundance is smaller than expected by about a factor of 5-10, further evidence that 

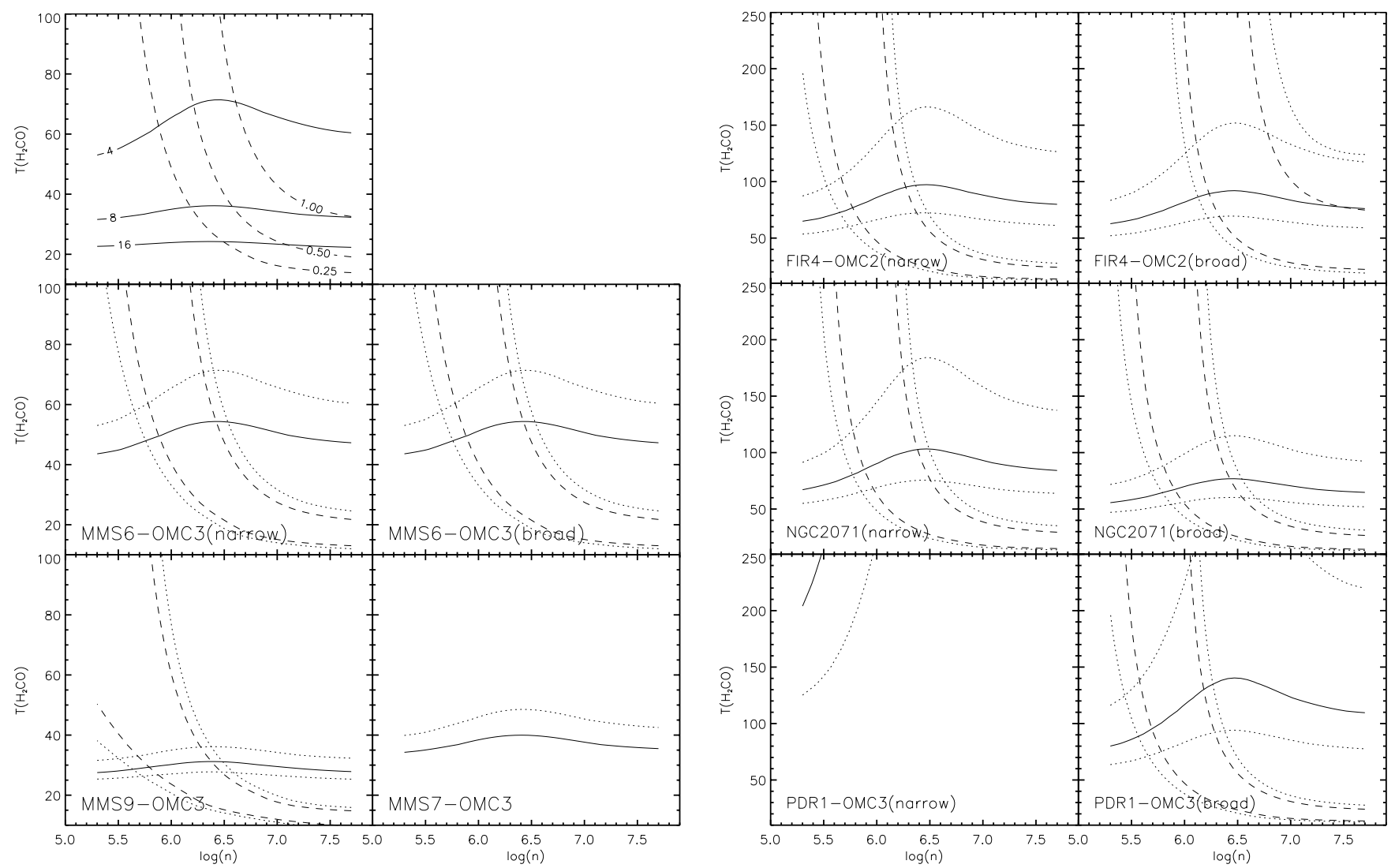

Fig. 5. Determination of the equilibrium physical properties of molecular gas from formaldehyde line ratios. The top left panel presents the expected line ratio for the $3_{03}-2_{02}$ and $3_{22}-2_{21}$ transitions as functions of temperature and density (solid contours) against the expected line ratio for the $5_{05}-4_{04}$ versus the $3_{03}-2_{02}$ transitions (dashed contours). The remaining panels show the range of physical properties obtainable for the sources in the present study, with the solid lines denoting the $3_{03}-2_{02}$ versus $3_{22}-2_{21}$ ratio and the dashed lines providing the location of the $5_{05}-4_{04}$ versus $3_{03}-2_{02}$ ratio. Two sets of dashed lines are shown to account for possible beam dilution (see text). Also, the dotted lines provide an indication of the range of uncertainty on the calculations.

almost all of the $\mathrm{CO}$ has been depleted onto grain surfaces in the dense envelopes. This result is in qualitative agreement with the more detailed modeling results presented by Jørgensen et al. (2002), where low-mass Class 0 and pre-stellar sources were observed to have similarly depleted $\mathrm{CO}$. Here, however, the outer envelope is warm, $T_{\mathrm{d}} \sim 30 \mathrm{~K}$ and the bulk of the depletion likely occurs in an intermediate zone between the hot, centrally heated deep interior and the externally heated surface layer. The higher abundance of CO in MMS9 is likely due to the smaller envelope, and thus a fractionally smaller intermediate zone. Detailed modeling of externally heated protostellar envelopes supports this hypothesis (Jørgensen et al., in prep.).

\subsection{Formaldehyde}

One of the advantages of $\mathrm{H}_{2} \mathrm{CO}$ is the ability to observe, in the same side-band of a single spectrum, two transitions which together probe the temperature of the gas under a large variation in density conditions. This feature removes many of the observational uncertainties inherent in sub-millimeter spectroscopy such as beam effects, weather conditions, and instrumental gain. The RADEX calculations for $\mathrm{H}_{2} \mathrm{CO}$ include energy levels up to $J=10$ and use collisional rate coefficients by Green (1991).
In the present study we observed three para-formaldehyde lines. Two of the lines were simultaneously observed in the $218 \mathrm{GHz}$ atmospheric window, $3_{03}-2_{02}$ and $3_{22}-2_{21}$. Because transitions between the different $K_{\mathrm{p}}$ ladders are radiatively forbidden, the ratio of the line strengths from transitions in different ladders depends only on the kinetic temperature of the gas, operating as a finely tuned thermometer over an extended density range (Mangum \& Wootten 1993). A third line was observed at $362 \mathrm{GHz}, 5_{05}-4_{04}$, within the same $K_{\mathrm{p}}$ ladder as the $3_{03}-2_{02}$ transition. Within a given $K_{\mathrm{p}}$ ladder the excitation energies do not vary dramatically and thus the ratio of these lines provides a useful density indicator at low to moderate densities $\left(n_{\mathrm{H}}<3 \times 10^{6} \mathrm{~cm}^{-3}\right)$ and a second temperature probe at higher densities. The first panel in Fig. 5 plots the expected line ratios against temperature and density.

The physical parameters derived from the formaldehyde lines are presented in Table 6, and are plotted graphically for each source in Fig. 5. The spread of line ratios $R_{33}$ and kinetic temperature of the gas inferred from the $3_{03}-2_{02}$ and $3_{22}-2_{21}$ transitions ranges from $R_{33}<2, T>300 \mathrm{~K}$, at the location of PDR1, to $R_{33}>10, T<30 \mathrm{~K}$ at the location of the sub-millimeter source MMS9. As expected, the intermediate mass star-forming regions NGC 2071 and FIR4 show moderately high temperatures $T>70 \mathrm{~K}$, consistent with the 
Table 6. Physical conditions and abundances discerned from formaldehyde.

\begin{tabular}{|c|c|c|c|c|c|c|c|c|}
\hline Source & $\mathrm{C}^{a}$ & $R_{33}^{b}$ & $R_{53}^{b}$ & $\begin{array}{c}T \\
(\mathrm{~K})\end{array}$ & $\begin{array}{c}n \\
\left(10^{6} \mathrm{~cm}^{-3}\right)\end{array}$ & $\begin{array}{c}N\left(\mathrm{H}_{2} \mathrm{CO}\right) \\
\left(10^{13} \mathrm{~cm}^{-2}\right)\end{array}$ & $\begin{array}{c}{\left[\mathrm{H}_{2} \mathrm{CO}\right] /[\mathrm{CO}]} \\
\left(10^{-5}\right)\end{array}$ & $\begin{array}{c}{\left[\mathrm{H}_{2} \mathrm{CO}\right] /\left[\mathrm{H}_{2}\right]} \\
\left(10^{-10}\right)\end{array}$ \\
\hline MMS6 & $\mathrm{n}$ & $>5$ & 0.6 & $<50$ & $1-3$ & 4.0 & & \\
\hline MMS6 & $\mathrm{b}$ & $>5$ & 0.6 & $<50$ & $1-3$ & 4.0 & & \\
\hline MMS6 & $\mathrm{t}$ & & & & & 8.0 & 1.7 & 0.8 \\
\hline MMS9 & $\mathrm{t}$ & 10 & 0.3 & 30 & $1-3$ & 2.2 & 0.8 & 1.6 \\
\hline MMS7 & $\mathrm{t}$ & $>7$ & - & $<40$ & - & 2.0 & & 0.9 \\
\hline FIR4 & $\mathrm{n}$ & 3.3 & 0.7 & 90 & $0.5-2$ & 12.0 & & \\
\hline FIR4 & $\mathrm{b}$ & 3.4 & 1.8 & 80 & $2-10$ & 20.0 & & \\
\hline FIR4 & $\mathrm{t}$ & & & & & 32.0 & 9.4 & 6.5 \\
\hline NGC 2071 & $\mathrm{n}$ & 3.2 & 0.9 & 90 & $1-3$ & 15.0 & & \\
\hline NGC 2071 & $\mathrm{~b}$ & 3.8 & 0.8 & 70 & $1-3$ & 17.0 & & \\
\hline NGC 2071 & $\mathrm{t}$ & & & & & 32.0 & 4.3 & 5.1 \\
\hline PDR1 & $\mathrm{n}$ & 1.8 & - & $>200$ & - & 0.8 & & \\
\hline PDR1 & $\mathrm{b}$ & 2.8 & 0.7 & 120 & $0.5-2$ & 6.0 & & \\
\hline PDR1 & $\mathrm{t}$ & & & & & 6.8 & 0.6 & 6.8 \\
\hline
\end{tabular}

${ }^{a}$ The letter designations refer to the velocity components with narrow (n), broad (b), and total (t).

${ }^{b} R_{33}$ is the ratio of the $3_{03}-2_{02}$ transition versus the $3_{22}-2_{21}$ transition and $R_{53}$ is the ratio of the $5_{05}-4_{04}$ transition versus the $3_{03}-2_{02}$ transition.

observability of infrared dust continuum emission. Mangum \& Wooten (1993) also observed FIR4 and NGC 2071, obtaining temperatures of $85 \mathrm{~K}$ and $80 \mathrm{~K}$ and with a range of probable temperatures bounding the results of this study. The only infrared source in OMC3, MMS7, shows no evidence for high temperatures.

The strength of the $5_{05}-4_{04}$ transition constrains the density of the environment from which the emission is observed. A complication arises from the difference in the beam size at the telescope for the $3_{03}-2_{02}$ and $5_{05}-4_{04}$ lines. If the line emission fills the larger $218 \mathrm{GHz}$ beam, then there are no difficulties in taking the ratio of the brightness temperatures of the two lines. However, if the source of the formaldehyde emission is smaller than the $362 \mathrm{GHz}$ beam the ratio of the beam sizes should be taken into account when comparing the brightness temperatures to account for the effect of beam dilution. Source sizes intermediate between the $218 \mathrm{GHz}$ and $363 \mathrm{GHz}$ beam require a varying normalization. Thus, the measured line ratio $R_{53}$, computed from the $5_{05}-4_{04}$ and $3_{03}-2_{02}$ lines, has an inherent uncertainty of $\sim 3$ due to the possible complications from intermediate beam dilution, with the value given in Table 6 strictly an upper limit.

For all sources the measured ratio $R_{53}$ is larger than 0.3 , implying a high density, $n>10^{6} \mathrm{~cm}^{-3}$. The relatively evolved far infrared source FIR4 shows two kinematically distinct components, a narrow-line region $\left(\Delta V \sim 1.5 \mathrm{~km} \mathrm{~s}^{-1}\right)$ requiring an effective density near $10^{6} \mathrm{~cm}^{-3}$ and broad-line component $(\Delta V \sim$ $6 \mathrm{~km} \mathrm{~s}^{-1}$ ) requiring a much higher density. While MMS6 and NGC 2071 also show distinct velocity components, the density does not obviously vary between the components for these sources. Intriguingly, in all three cases the temperature deduced from both the narrow and broad-line components are quite similar.

With the temperature and density estimated, the column density of para-formaldehyde can be deduced from the integrated line strength of the $3_{03}-2_{02}$ transition. The high temperature equilibrium ratio of the para to ortho forms (1:3) was used to convert this result into a total formaldehyde column density. The inferred abundance of formaldehyde was then measured against both the $\mathrm{H}_{2}$ column, deduced from the submillimeter dust continuum emission, and the measured $\mathrm{CO}$ column. The results are presented in Table 6. The two infrared sources show the largest abundance of formaldehyde measured against the $\mathrm{CO}$. For these sources the abundance ratios lie in the range $\left[\mathrm{H}_{2} \mathrm{CO}\right] /[\mathrm{CO}]=2.5-10.0 \times 10^{-5}$. The submillimeter sources MMS6 and MMS9 show a lower abundance ratio $\left[\mathrm{H}_{2} \mathrm{CO}\right] /[\mathrm{CO}] \sim 1 \times 10^{-5}$. Disentangling the importance of $\mathrm{CO}$ depletion in these data complicates any analysis unless the formaldehyde depletes in a similar fashion to $\mathrm{CO}$. However, comparing the formaldehyde abundance to $\mathrm{H}_{2}$ produces a clear relation (see Table 6 and Fig. 8). The hot PDR region and the infrared sources, NGC 2071 and FIR4, have the largest formaldehyde abundance, approximately 5-10 times greater than observed for the sub-millimeter sources. At least half of the increase in the abundance of formaldehyde in the two infrared sources is due to the broad-line component.

In low-mass Class 0 sources, typical $\left[\mathrm{H}_{2} \mathrm{CO}\right] /[\mathrm{CO}]$ abundance ratios in the outer region are a few $\times 10^{-5}$, similar to those observed here, but jumps in the $\mathrm{H}_{2} \mathrm{CO}$ abundance by more than a factor of 100 have been found in the inner warm envelopes, consistent with ice evaporation (e.g., Ceccarelli et al. 2000; Schöier et al. 2002; Maret et al., in prep.). $\mathrm{H}_{2} \mathrm{CO}$ is 
expected to be a product of grain surface hydrogenation of $\mathrm{CO}$ and has been tentatively detected in interstellar ices with an abundance of a few percent with respect to $\mathrm{H}_{2} \mathrm{O}$ ice, corresponding to $\sim 10^{-6}$ with respect to $\mathrm{H}_{2}$ (Keane et al. 2001b). Thus, the trend observed here for the protostellar sources could be consistent with evaporation of $\mathrm{H}_{2} \mathrm{CO}$-containing ices in the warm interior regions, with more energetic sources containing a larger fractional mass of envelope at high enough temperatures to evaporate such ices. For high-mass sources, $\mathrm{H}_{2} \mathrm{CO}$ shows a less clear relation with temperature (van der Tak et al. 2000b).

\subsection{Methanol}

Methanol emission lines have been detected in complex bands at 241 and $338 \mathrm{GHz}$, with excitation energies ranging upward from $T_{\mathrm{ex}} \sim 30 \mathrm{~K}$. The detection of many $\mathrm{CH}_{3} \mathrm{OH}$ lines in our spectra allows for the construction of a rotation diagram for each source in order to get a first indication of the temperature and column density. Described in detail by Blake et al. (1987), this method assumes that the lines are optically thin and that the excitation can be characterized by a single temperature. The resulting rotation diagrams, however, are often misleading. Direct computation of the level populations using RADEX shows that the higher excitation lines require increasingly larger critical densities and thus the derived temperature from rotation diagram fitting is heavily dependent on the density of the medium in which the methanol resides (see also the discussion by Bachiller et al. 1995). Figure 6 reveals the effect on the rotation diagram produced by increasing the critical density of the medium while holding the kinetic temperature constant at $100 \mathrm{~K}$ and the total column density at $N_{\mathrm{CH}_{3} \mathrm{OH}}=$ $10^{14} \mathrm{~cm}^{-3}$. Even though reasonable linear fits are produced, the derived temperature ranges from $25 \mathrm{~K}$ for $n_{\mathrm{H}_{2}}=10^{7} \mathrm{~cm}^{-3}$ to $80 \mathrm{~K}$ for $n_{\mathrm{H}_{2}}=10^{9} \mathrm{~cm}^{-3}$. The derived column densities also overestimate the actual amount of methanol by factors of a few.

For a protostellar source with both density and temperature gradients a proper analysis becomes even more complex. Given that higher excitation lines tend to have higher critical densities, it is likely that these lines are dominated by emission solely from the deep interior of the envelope. Indeed, there is some evidence that the source size decreases as the required excitation of the line increases (Hatchell et al. 1998). The bulk properties of the envelope derived from methanol, however, are expected to be only slightly weighted toward the highest density and temperature regions due to the small angular extent of these extreme locations. Complicating even a detailed analysis, there is evidence for an enhanced abundance of methanol at high temperatures, $T>90 \mathrm{~K}$, in protostellar envelopes (van der Tak et al. 2002b; Schöier et al. 2002).

Despite the above concerns, one might hope that methanol may be used both as a temperature and density indicator by treating all of the lines through a proper statistical equilibrium calculation. The RADEX calculations for methanol include energy levels up to $260 \mathrm{~K}$ and use collisional rate coefficients by Lees \& Haque (1974) and Walmsley (private communication).

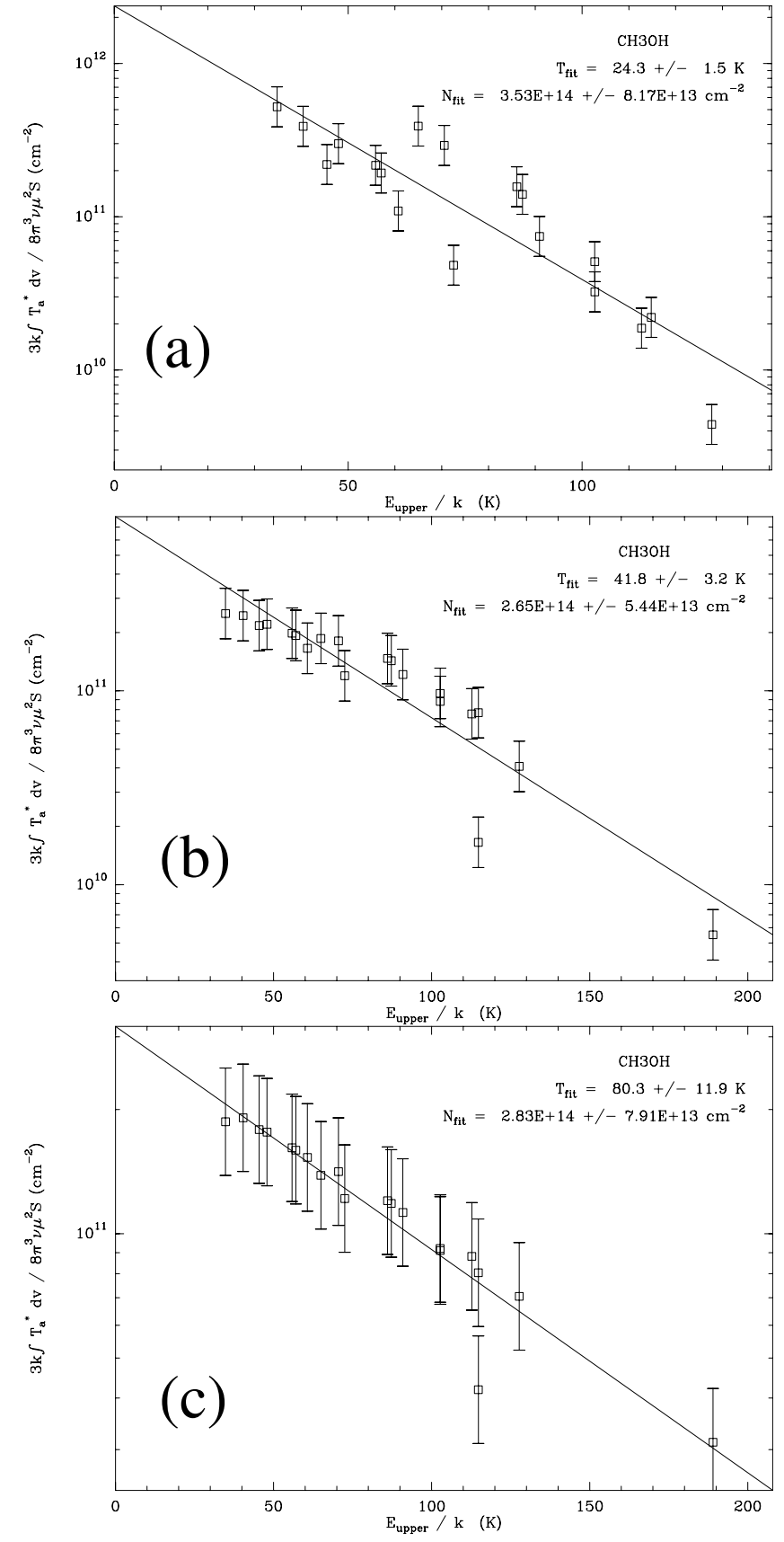

Fig. 6. Rotation diagrams for methanol. The input data are taken from the result of RADEX equilibrium calculations at a temperature of $100 \mathrm{~K}$. a) Calculation with $n=10^{7} \mathrm{~cm}^{-3} . .2$ The slope of the data at high energies is significantly affected by the sub-thermal excitation, and the derived temperature from the rotation diagram does not correspond to the physical condition. The low energy lines are much closer to being thermalized, however, and the column density derived is close to the input column density of $10^{14} \mathrm{~cm}^{-3}$. b) Calculation with $n=10^{8} \mathrm{~cm}^{-3}$. The slope of the data at high energies is still affected by the sub-thermal excitation, and the derived temperature from the rotation diagram continues to underestimate the physical condition. c) Calculation with $n=10^{9} \mathrm{~cm}^{-3}$. At this density the rotation diagram produces a reasonable fit to the input physical conditions.

For this paper, all the measured methanol lines were compared with RADEX computations to produce a chi-squared fit as a 
Table 7. Physical conditions and abundances discerned from methanol.

\begin{tabular}{lcccccc}
\hline \hline Source & $\mathrm{C}^{a}$ & $\begin{array}{c}T \\
(\mathrm{~K})\end{array}$ & $\begin{array}{c}n \\
\left(10^{7} \mathrm{~cm}^{-3}\right)\end{array}$ & $\begin{array}{c}N\left(\mathrm{CH}_{3} \mathrm{OH}\right) \\
\left(10^{13} \mathrm{~cm}^{-2}\right)\end{array}$ & $\begin{array}{c}{\left[\mathrm{CH}_{3} \mathrm{OH}\right] /[\mathrm{CO}]} \\
\left(10^{-5}\right)\end{array}$ & $\begin{array}{c}{\left[\mathrm{CH}_{3} \mathrm{OH}\right] /\left[\mathrm{H}_{2}\right]} \\
\left(10^{-10}\right)\end{array}$ \\
\hline MMS6 & $\mathrm{t}$ & 50 & 1.0 & 3.0 & 0.7 & 0.3 \\
MMS9 & $\mathrm{t}$ & $<30$ & 0.5 & 1.5 & 0.6 & 1.1 \\
FIR4 & $\mathrm{n}$ & 70 & $>10$ & 30 & & 6.1 \\
FIR4 & $\mathrm{b}$ & 80 & 5 & 95 & & 19 \\
FIR4 & $\mathrm{t}$ & & & 125 & 37 & 25 \\
NGC 2071 & $\mathrm{t}$ & 60 & 0.5 & 30 & 5.6 & 5.1 \\
& & & & & & 5.8 \\
PDR1 & $\mathrm{t}$ & $>100$ & 5.0 & 6.0 & 0.6 & \\
\hline
\end{tabular}

${ }^{a}$ The letter designations refer to the velocity components with narrow (n), broad (b), and total (t).

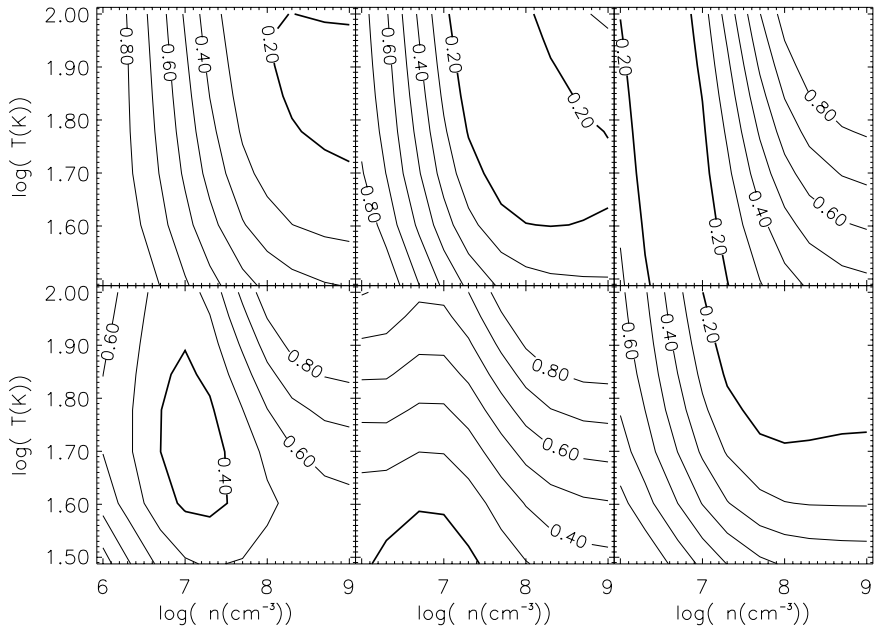

Fig. 7. Chi-squared confidence fits to the physical parameters of sources using the observed methanol lines and the radiative transfer code RADEX. The sources are (Top Left) FIR4 narrow component, (Top Center) FIR4 broad component, (Top Right) NGC 2071, (Bottom Left) MMS6, (Bottom Center) MMS9, (Bottom Right) PDR1. Note that there is often a strong degeneracy between the best fit temperature and density due to the lack of thermalization of the higher energy transitions.

function of temperature and density. The results are presented graphically in Fig. 7 and are tabulated in Table 7. For FIR4, the methanol measurements can be separated into narrow and broad line components. The narrow line region requires very high density and temperature $n>10^{8} \mathrm{~cm}^{-3}, T \sim 70 \mathrm{~K}$, while the broad line component requires $n \sim 10^{8} \mathrm{~cm}^{-3}, T \sim 80 \mathrm{~K}$. The broad line region appears to be beam diluted, at least with respect to the larger $241 \mathrm{GHz}$ beam, requiring a normalization factor between the two sets of measurements to produce reasonable chi-squared fits. These results are not consistent with the formaldehyde measurements in that higher densities are required to account for the methanol observations. Given a range of densities within the surroundings of FIR4, however, the methanol observations would be most sensitive to the highest density gas. In contrast, the other infrared source, NGC 2071, is best fit by a moderate density, $n \sim 5 \times 10^{6} \mathrm{~cm}^{-3}$, and a moderate temperature, $T \sim 60 \mathrm{~K}$, consistent with the formaldehyde measurements.

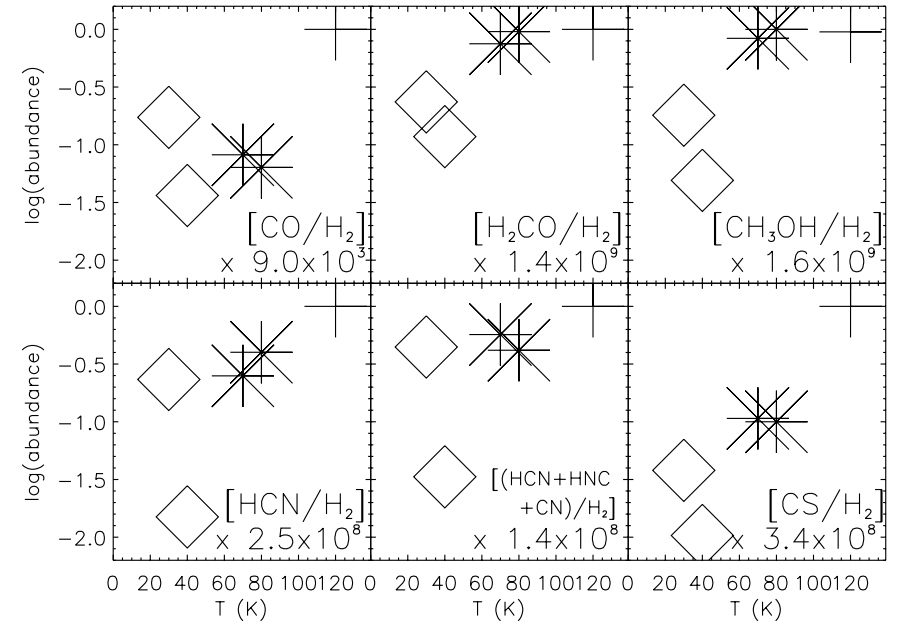

Fig. 8. The range in abundances of the various molecular species investigated against $T\left(\mathrm{H}_{2} \mathrm{CO}\right)$. The abundances have been derived assuming a constant temperature and density envelope. Diamonds represent sub-millimeter sources, stars represent infrared sources, and the cross represents the PDR, with symbol size approximating the order unity uncertainty expected in the measurements. For all protostellar sources the $\mathrm{CO}$ abundance is depleted by about an order of magnitude. For all species the infrared sources show a remarkable similarity in abundances, however, in the case of methanol the very broad $>10 \mathrm{~km} \mathrm{~s}^{-1}$ component from FIR4 has not been included. For most molecules the sub-millimeter sources have lower abundances while the PDR has the highest abundance.

The two sub-millimeter sources show some variation in the inferred temperature. MMS6 requires a moderate temperature $T \sim 50 \mathrm{~K}$ while MMS9 requires a low temperature $T<30 \mathrm{~K}$. For both sources, the methanol appears to probe a dense inner region, $\sim 10^{7} \mathrm{~cm}^{-3}$ compared with the formaldehyde derived envelope densities $\sim 10^{6} \mathrm{~cm}^{-3}$. This is likely due to the higher critical density required to excite the methanol transitions. Across all the sources there is a reasonable correlation between the methanol and formaldehyde temperatures with the sub-millimeter sources being the coldest, the infrared sources being moderately warm, and the PDR having the highest temperatures.

The derived abundance of methanol is similar to that of formaldehyde. The warm infrared sources have the highest 
Table 8. Abundances discerned from $\mathrm{HCN}, \mathrm{HNC}$, and $\mathrm{CN}$.

\begin{tabular}{lcccccc}
\hline \hline Source & {$[\mathrm{HCN}] /[\mathrm{HNC}]$} & $\begin{array}{c}N(\mathrm{HCN}) \\
\left(10^{14} \mathrm{~cm}^{-2}\right)\end{array}$ & $\begin{array}{c}N(\mathrm{CN}) \\
\left(10^{14} \mathrm{~cm}^{-2}\right)\end{array}$ & $\begin{array}{c}{[\mathrm{HCN}] /[\mathrm{CN}]} \\
\left(10^{0}\right)\end{array}$ & $\begin{array}{c}{[\mathrm{HCN}] /\left[\mathrm{H}_{2}\right]} \\
\left(10^{-10}\right)\end{array}$ & $\begin{array}{c}{[\mathrm{CN}(\text { total })] /\left[\mathrm{H}_{2}\right]} \\
\left(10^{-10}\right)\end{array}$ \\
\hline MMS6 & 6.3 & 0.6 & $<2.0$ & $>0.3$ & 0.6 & $<2.4$ \\
MMS9 & 5.4 & 1.3 & 2.8 & 0.5 & 9.3 & 32. \\
FIR4 & 11. & 8.0 & 6.0 & 1.3 & 16. & 30. \\
NGC 2071 & 6.0 & 6.4 & 19. & 0.3 & 10. & 41. \\
PDR1 & 33. & 4.0 & 2.0 & 2.0 & 40. & 72. \\
\hline
\end{tabular}

abundance with respect to CO. However, when compared with the total column of hydrogen deduced from the sub-millimeter continuum measurements (Table 5), the PDR region also shows an enhanced abundance of methanol. Considering that the methanol observations are predominantly probing higher density regions within each envelope, it is likely that these abundance estimates are strictly lower limits. Differences in the abundance derivations across sources may be affected by the extent of the warm interior within each envelope.

Like $\mathrm{H}_{2} \mathrm{CO}, \mathrm{CH}_{3} \mathrm{OH}$ is expected to be a product of the hydrogenation of CO on grain surfaces (Tielens \& Charnley 1997) and is detected in grain mantles, albeit with significant variations between different sources (Dartois et al. 1999). $\mathrm{CH}_{3} \mathrm{OH}$ abundance jumps of at least a factor of 100 have been found in various high-mass objects (van der Tak et al. 2000b), whereas the well-studied low-mass Class 0 source IRAS 16293-2422 shows a jump by nearly three orders of magnitude in the inner warm envelope (Schöier et al. 2002). Such high $\mathrm{CH}_{3} \mathrm{OH}$ abundances can only be produced by evaporation from grain mantles. Enhanced $\mathrm{CH}_{3} \mathrm{OH}$ abundances have also been detected in outflows at positions offset from the protostar, e.g., in L1157 (Bachiller \& Pérez-Gutiérrez 1997), where the $\mathrm{CH}_{3} \mathrm{OH}$ is released from the grain mantles by shocks rather than thermal evaporation. The general trend of an increased $\mathrm{CH}_{3} \mathrm{OH}$ abundance with temperature observed for our sources is consistent with this picture. The presence of both narrow and broad $\mathrm{CH}_{3} \mathrm{OH}$ line profiles suggests that both evaporation mechanisms play a role in some objects (e.g., NGC 2071).

\subsection{Nitrogen-bearing species}

\subsection{1. $\mathrm{HCN}$ and $\mathrm{HNC}$}

Identical lines of both $\mathrm{HCN}$ and HNC are observed simultaneously with a single spectrograph setting at the JCMT and thus, assuming the collisional cross sections for the various excitations are identical across species, the relative abundance of the two molecules can be simply determined if the transitions are optically thin. To test the optical depth conditions, the ratios of line strengths between $\mathrm{HCN}$ and $\mathrm{H}^{13} \mathrm{CN}$, and $\mathrm{HNC}$ and $\mathrm{HN}^{13} \mathrm{C}$ were computed. In all cases the $\mathrm{HCN}$ line was found to be moderately to strongly optically thick $\left(\tau_{\mathrm{HCN}} \sim 3-6\right)$ however, only the most energetic sources FIR4 and NGC 2071 were found to have optically thick HNC lines $\left(\tau_{\mathrm{HNC}} \sim 2-3\right)$.
The $[\mathrm{HCN}] /[\mathrm{HNC}]$ ratio tabulated in Table 8 was determined by accounting for these optical depth effects.

For the four protostellar regions observed, the integrated $[\mathrm{HCN}] /[\mathrm{HNC}]$ abundance ratio ranges from 5-10. Using the chemical models by Schilke et al. (1992), this would imply low temperatures $T<30 \mathrm{~K}$ and high densities $n>10^{7} \mathrm{~cm}^{-3}$, or high temperatures $T>50 \mathrm{~K}$ and moderate to low densities $n<10^{6} \mathrm{~cm}^{-3}$. For PDR1, the ratio rises to 70 , requiring both high densities $n>10^{6} \mathrm{~cm}^{-3}$ and high temperatures $T>50 \mathrm{~K}$ (Schilke et al. 1992). It is worth noting that the main observational difference between the least energetic sub-millimeter sources, MMS6 and MMS9, and the most energetic sources, FIR4 and NGC 2071, is in the peak brightness of the lines. Thus, it would appear that the conditions probed by the HCN and HNC molecules in all four sources are nearly identical but that the solid angle covered in the most energetic sources is somewhat larger. RADEX calculations support this hypothesis. For the determined physical conditions of the optically thick HCN lines the excitation temperature is expected to be relatively large $T_{\mathrm{ex}}>10 \mathrm{~K}$. The least energetic sources have observed peak intensities of only $T_{\text {peak }} \sim 1 \mathrm{~K}$, while the infrared sources show $T_{\text {peak }} \sim 15 \mathrm{~K}$. Care should be taken in using chemistry to constrain temperature, however, since the lowmass sources observed by Jørgensen et al. (2003; in prep.) have high $\mathrm{HCN} / \mathrm{HNC}$ abundance ratios at low temperatures, which are not readily explained by the Schilke et al. (1992) models.

RADEX calculations for HCN, including energy levels up to $J=12$ and using collisional coefficients by Green \& Thaddeus (1974), have been performed to derive HCN abundances using the estimated physical conditions derived from formaldehyde. The resulting abundances with respect to $\mathrm{CO}$ are $\sim 5 \times 10^{-5}$ for the sub-millimeter sources and PDR1; however, the production of $\mathrm{HCN}$ appears to be significantly enhanced in the far infrared sources FIR4 and NGC 2071. The HCN abundances with respect to $\mathrm{H}_{2}$ vary from $0.6 \times 10^{-10}$ to $40 \times 10^{-10}$ (Table 8). These latter abundances show a clear trend with increasing source energetics. HCN has not been detected in interstellar ices due to a lack of clear spectroscopic signatures, but is expected to freeze-out in cold regions like most molecules. The $\mathrm{HCN}$ abundance is known to be enhanced by orders of magnitude in the warm inner regions of massive protostars (Lahuis \& van Dishoeck 2000; Boonman et al. 2001), but the high temperature routes to HCN do not become effective until $T>200 \mathrm{~K}$ (e.g., Doty et al. 2002). It remains to be determined whether the intermediate mass sources studied here have sufficient amounts 
Table 9. Physical conditions and abundances discerned from CS and SO.

\begin{tabular}{lcccccc}
\hline \hline Source & $\begin{array}{c}N(\mathrm{CS}) \\
\left(10^{13} \mathrm{~cm}^{-2}\right)\end{array}$ & $\begin{array}{c}{[\mathrm{CS}] /\left[\mathrm{H}_{2}\right]} \\
\left(10^{-10}\right)\end{array}$ & $\begin{array}{c}T_{\mathrm{CS}} \\
(\mathrm{K})\end{array}$ & $\begin{array}{c}N(\mathrm{SO}) \\
\left(10^{14} \mathrm{~cm}^{-2}\right)\end{array}$ & $\begin{array}{c}{[\mathrm{SO}] /\left[\mathrm{H}_{2}\right]} \\
\left(10^{-10}\right)\end{array}$ & $\begin{array}{c}{[\mathrm{SO}] /[\mathrm{CS}]} \\
\left(10^{0}\right)\end{array}$ \\
\hline MMS6 & 3.5 & 0.3 & $>50$ & $<2.6$ & $<2.5$ & $<7.4$ \\
MMS9 & 1.5 & 1.1 & & & & $<2.9$ \\
FIR4 & 14. & 2.9 & $>100$ & $<4.0$ & $<8.2$ & $<2.0$ \\
NGC 2071 & 19. & 3.1 & $>50$ & & & \\
PDR1 & 29. & 29. & $>50$ & 0.8 & 8.0 & 0.3 \\
\hline
\end{tabular}

of gas at such high temperatures to enhance $\mathrm{HCN}$ significantly. The trends observed here are more likely due to general evaporation of ices with temperatures between 20 and $100 \mathrm{~K}$, enhancing the overall gas-phase abundances.

Detailed consideration of the HCN line shows that it is usually wider than the HNC line. This requires that in the broad line region the ratio of the two molecules is much higher than the ratio of the total integrated intensities, implying higher temperatures and/or higher densities if chemical equilibrium is assumed (Schilke et al. 1992). Another possible explanation for the general lack of broad line HNC may be found in the shock model solution of Schilke et al. (1992) where HNC is preferentially destroyed within the interaction region, reforming downstream from the shock front. In particular, the broad HCN emission, coupled with little or no HNC emission, found in the shock knot SK1 is likely best explained in this manner. Interferometer data of the low-mass source IRAS 16293-2422 also show that HNC avoids the warm inner region, in contrast with HCN (Schöier et al. 2003; in prep.).

\subsection{2. $\mathrm{CN}$}

Observations of the $\mathrm{CN}$ hyperfine structure at $340 \mathrm{GHz}$ provides a set of constraints for the modeling of $\mathrm{CN}$ (see Table 3 ). In the optically thin limit, the ratio between the strongest and weakest hyperfine components should be $\sim 15$; however, this is only observed in the spectrum of PDR1. In all other regions, except possibly MMS6, the main CN component is optically thick, $\left(\tau_{\mathrm{CN}} \sim 3-6\right)$, while the weaker components remain reasonably optically thin. Assuming that the line flux is predominantly from the outer envelope surrounding the sources, the column of $\mathrm{CN}$ derived from the optically thin hyperfine lines is presented in Table 8. The RADEX calculations for $\mathrm{CN}$ include energy levels up to $J=15$. As found for the abundance of HCN, only the coldest, least energetic source, MMS6 has a dramatically low abundance of $\mathrm{CN}$. However, a clear correlation is found between the ratio $[\mathrm{HCN}] /[\mathrm{HNC}]$ and $[\mathrm{HCN}] /[\mathrm{CN}]$. Assuming that all $\mathrm{CN}$ is locked up in these three molecules, the total abundance of $\mathrm{CN}$ can be readily determined. Table 8 displays the results of this calculation. With the exception of the sub-millimeter source (MMS6) the derived total abundance of $\mathrm{CN}$ is much more constant than any of the individual molecular species.

Results of systematic studies of $\mathrm{CN}$ in low- or highmass sources are not yet available, but like $\mathrm{HNC}, \mathrm{CN}$ seems to avoid the inner warm region in the low-mass protostar IRAS 16293-2422. The radical is known to be abundant in the outer regions of PDRs (e.g., Fuente et al. 1998), where it is produced by rapid gas-phase chemistry of $\mathrm{CH}$ and $\mathrm{C}_{2}$ with N. Alternatively, it can be a photodissociation product of $\mathrm{HCN}$. Photodissociation of $\mathrm{CN}$ itself requires photons with energies higher than $12 \mathrm{eV}$. The observed lack of variation of the $\mathrm{CN}$ abundance in the protostellar sources observed here implies that they are exposed to similar radiation fields. As mentioned earlier, the Orion region is bathed in a significantly increased external radiation field due to the close proximity of massive stars. The much lower abundance within the massive envelope of MMS6, however, is not easily explained via this hypothesis unless the structure of the envelope is such that the interstellar radiation field is unable to penetrate through the surface layer and into the significant interior.

\subsection{Sulphur-bearing species}

\subsubsection{CS}

Both $\mathrm{CS}$ and the rarer $\mathrm{C}^{34} \mathrm{~S}$ isotope were observed in the $J=5-4$ transition. In all cases the line ratio was less than the isotopic ratio of 22 implying moderately optically thick conditions within the CS line but optically thin conditions for the isotope. Taking the formaldehyde physical conditions for the temperature and the density, the column density of CS was determined using RADEX (Table 9). The RADEX calculations for CS include energy levels up to $J=12$. The abundance with respect to $\mathrm{H}_{2}$ trends with the source energetics, rising from a few $10^{-11}$ to a few $10^{-10}$, in the protostellar sources. This is a stronger trend than seen for HCN suggesting that the more energetic sources are providing more heating to their outer envelope. The abundance rises sharply to a few $10^{-9}$, in the warm PDR1 source.

For four of the sources the $J=7-6$ transition of $C^{34} \mathrm{~S}$ was also detected. In all observed cases the ratio of the $J=7-6$ versus $J=5-4$ lines implies a warm, $(T>50 \mathrm{~K})$ and dense $\left(n>10^{6}\right)$ environment (Table 9). The $\mathrm{C}^{34} \mathrm{~S}$-derived temperature is higher than that derived from $\mathrm{H}_{2} \mathrm{CO}$ and more likely refers to the inner warm and dense region rather than the outer envelope. If more transitions are observed, a detailed density structure for each of the sources could be determined. 


\subsubsection{SO}

The only observed SO transition was unfortunately blended with a methanol line and thus difficult to accurately measure. Despite this complication, three sources, spanning a range of energetics and conditions, have unambiguous detections for SO (MMS6, NGC 2071, and PDR1). Assuming the formaldehyde derived properties for the physical conditions of the gas, RADEX was used to determine the required column density of SO for each of the sources, The RADEX calculations for SO include energy levels up to $580 \mathrm{~K}$. The results are presented in Table 9, along with the abundance of SO in each source and the SO to CS abundance ratio.

Observations of more SO lines and other sulphur-bearing species, such as $\mathrm{SO}_{2}$ and $\mathrm{H}_{2} \mathrm{~S}$, are needed to study possible differences in the sulphur-chemistry in these sources.

\section{Discussion and conclusion}

This molecular line study was undertaken to determine if morphological clues and qualitative indicators were observable across a range of environmental conditions, from pre-stellar and young protostellar envelopes $L_{\mathrm{bol}} \sim 1-100 L_{\odot}$ through infrared bright energetic sources $L_{\text {bol }} \sim 400 L_{\odot}$, a PDR knot and a shock front. Several trends are apparent, especially in the derived abundances of many molecular species. As well, there are a number of spectral hints that protostellar sources reside within the sub-millimeter clumps, excluding the PDR and the shock knot.

\subsection{Abundances}

Despite the inherent danger in assuming a single temperature and density for the environments of each source, general trends in abundance are observed. It is worth noting that detailed modeling (van der Tak et al. 2000a; Schöier et al. 2002; Doty et al. 2002; Jørgensen et al. 2002) provides a much more accurate determination of relative abundances, especially for molecules which are excited only in parts of the envelope. Also, the presence of abundance gradients or "jumps" can be established for some molecules. Such models require a determination of the density and temperature profiles of the sources from the dust continuum emission, which has not yet been completed for this study. Schöier et al. (2002) show that the inferred abundances using a constant temperature and density do not differ by more than a factor of a few from the detailed analysis as long as the adopted conditions are appropriate for the particular molecule or line, which should be the case for this work. However, detailed modeling requires significant input as to the density and temperature profile of the source, as well as relying on additional assumptions such as the dust emissivity profile. Additionally, the Orion sources are bathed in a strong external radiation field ( $\mathrm{Li}$ et al. 2003) requiring careful consideration of the exterior conditions of each source envelope where the dust and gas temperatures reach $T \sim 30 \mathrm{~K}$. This study is concerned primarily with the constraints on physical conditions provided by the variations in the molecular tracers without resorting to detailed modeling of individual sources.
Some strong trends are observed across the source list. The peak brightness and integrated line strength of both $\mathrm{CO}$ and ${ }^{13} \mathrm{CO} 3-2$ lines follow the energetics and warm dust temperatures of the envelopes. Despite the observed depletion of CO in the protostellar source envelopes (typically the abundance is a factor of 10 lower than the mean molecular cloud value), in all cases the objects are visible in each of the observed $\mathrm{CO}$ lines.

The formaldehyde derived temperatures, $T\left(\mathrm{H}_{2} \mathrm{CO}\right)$, do not match the envelope dust temperatures, measured in the submillimeter. However, for the sub-millimeter sources MMS6, MMS7, and MMS9 $T\left(\mathrm{H}_{2} \mathrm{CO}\right)$ is consistent with the gas temperature in the outer envelope as seen in the ${ }^{13} \mathrm{CO}$ line. For the infrared sources, FIR4 and NGC 2071, T( $\left.\mathrm{H}_{2} \mathrm{CO}\right)$ requires internal heating through a significant fraction of each envelope. Detailed modeling of other sources provides evidence that the formaldehyde abundance increases in the warm, dense interiors of protostellar envelopes, and that grain mantle evaporation may be important in producing the enhancement. However, the relatively low lying lines observed in this study are more accurate tracers of the extended envelope conditions, where most of the mass resides (Jørgensen et al., in prep.). While more uncertain, the methanol derived temperatures follow the same trend as the formaldehyde temperatures. For the remainder of the discussion we adopt the formaldehyde conditions as representative of the bulk conditions within each source.

Despite uncertainties of order unity, the abundance of both formaldehyde and methanol correlate well against the source energetics (Fig. 8). The changing abundance may reflect jump conditions in formaldehyde and methanol abundance with dust temperature in the inner warm part. Ignoring the broad (outflow) methanol component in NGC 2071, the abundance of both formaldehyde and methanol may be plotted as a step function with cold sources, $T<50 \mathrm{~K}$, having a low abundance and warm sources, $T>50 \mathrm{~K}$, having about five times higher abundance. Although less observational data from the study are available, the CS abundance also appears to correlate with source energetics.

The abundance of $\mathrm{HCN}$ is also sensitive to source conditions; however, this is mostly determined by extreme cases MMS6 and PDR1. In contrast to the other observed species, little variation of abundance was found for HCN among the energetic sources, FIR4 and NGC 2071, and the weak submillimeter source MMS9. Considering the three dominant $\mathrm{CN}$ bearing species ( $\mathrm{HCN}, \mathrm{HNC}$, and $\mathrm{CN}$ ), the total abundance of these three molecules is quite constant across a wide range of conditions, varying by less than thirty percent between MMS9, FIR4, and NGC 2071 and only varying by a factor of two when the PDR region is included. Only the cold, dense submillimeter source MMS6 appears to have a severely depleted abundance of CN-bearing species. The enhanced external radiation field in Orion may be responsible for this apparent equilibrium.

The general observed trend of increased abundance with source energetics is consistent with a scenario in which freezeout of molecules occurs in the cooler intermediate zone within the envelope and evaporation of ices occurs in the warmer interior and possibly exterior regions. Similar trends have been observed for samples of low-mass (Jørgensen et al. 2002, 2003) 
and high-mass (van der Tak et al. 2000a,b; Boonman et al. 2003) sources without external heating, although not for all molecules. The only other intermediate-mass source that has been studied in some detail chemically is AFGL 490 (Schreyer et al. 2002) which has a luminosity of $10^{3} L_{\odot}$. This source has a large envelope, with abundances comparable to those of the warmer sources observed here. Strong solid-state features of various species indicative of freeze-out are also detected.

None of the observed sources shows the characteristic crowded line spectra of a "hot core", such as found for highmass protostars like G34.3+0.15 (Macdonald et al. 1996), G327.3-0.6 (Gibb et al. 2000) or $\mathrm{W} 3\left(\mathrm{H}_{2} \mathrm{O}\right)$ (Helmich \& van Dishoeck 1997). Deeper integrations are needed to determine whether complex organic molecules like $\mathrm{CH}_{3} \mathrm{OCH}_{3}$ are present in the warmer sources in our sample, e.g. FIR4 or NGC 2071. These molecules are expected to be produced by gas-phase chemistry between evaporated ices, but have so far only been observed in high-mass sources. The different dynamical time scales of the hot core gas in low- versus high-mass objects compared with the chemical timescales of $\sim 10^{4} \mathrm{yr}$ may prevent formation of such second generation species.

The PDR1 position differs chemically from the other sources by having the highest gas-phase abundances overall and the largest $[\mathrm{HCN}] /[\mathrm{HNC}]$ ratio. PDRs also generally have high abundances of radicals such as $\mathrm{CN}$ and $\mathrm{C}_{2} \mathrm{H}$ and ions like $\mathrm{CO}^{+}$(Jansen et al. 1995). Indeed, a study of a set of more evolved intermediate-mass sources by Fuente et al. (1998) has identified $\mathrm{CN}$ as a particularly good diagnostic of the ultraviolet radiation. In our sources, this trend is not so evident, likely because all sources are located in the Orion region which is bathed in intense radiation from nearby $\mathrm{O}$ and $\mathrm{B}$ stars.

Few molecules are observed in the SK1-OMC3 shock and only $\mathrm{CO}$ shows truly broad line wings at that position. Sulphurcontaining species like $\mathrm{H}_{2} \mathrm{~S}, \mathrm{SO}$ and $\mathrm{SO}_{2}$ are predicted to be abundant in shock models (e.g., Leen \& Graff 1988) and are seen to be enhanced in the Orion-KL plateau gas with broad line wings, but are not prominent here; however, the source is quite weak and the lines may be below our detection limits. In NGC 2071, molecules like $\mathrm{CH}_{3} \mathrm{OH}$ are present in the outflow but this results from grain evaporation in the shock rather than high-temperature chemistry. Chernin et al. (1994) have observed broad SO emission in NGC 2071 with some abundance enhancement. Deeper searches for shock diagnostics and accurate determinations of abundances at shocked and non-shocked positions in these and other sources are needed.

\subsection{Protostellar source diagnostics}

A large number of sub-millimeter continuum surveys have now been completed in star-forming molecular clouds, and hundreds of new sub-millimeter bright envelopes have been enumerated. However, an outstanding question is which fraction of these objects surround protostellar sources and which are pre-stellar. Within the present study there were no clear prestellar objects although MMS6 appear somewhat ambiguously defined as a Class 0 source. Despite this lack of an obvious pre-stellar candidate, it is worth considering if any of the observed molecular signatures require an embedded heating source.

For many of the sub-millimeter objects found within surveys, ${ }^{13} \mathrm{CO}$ observations have provided no indication of a coincident CO peak (Mitchell et al. 2001). Thus, the clear measurement of $\mathrm{CO}$ isotopes in this sample, despite the depletion, may provide a clue to which objects contain embedded sources, perhaps by warming and evaporating $\mathrm{CO}$ back into the environment from which it had frozen out during an earlier epoch. Alternatively, the presence of a strong external radiation field may bias the results in Orion. The broad CO line wings provide clear evidence of enhanced kinematics within these condensations and, along with the ubiquitous outflows in star-forming regions, act as a sign post for embedded sources.

The appearance of molecular lines with relatively high excitation temperatures, and preferably also with high critical densities such that the warm region might be inferred to be deeper within the envelope, should provide a signature to Class 0 and later sources. Thus, the methanol lines are likely the strongest indicator of a warm, dense region within the envelope. For all sources observed in this study, except the known outflow source MMS9, the required internal temperature deduced from the methanol lines is $>50 \mathrm{~K}$, a temperature unattainable without an energetic internal source. The CS 7-6 line also provides evidence of a warm, dense interior region in all sources except MMS9. It is interesting to note that all the protostellar sources considered in this paper require high densities in their interior. Standard models for star formation expect a power-law density distribution toward the clump center during the collapse phase (Shu 1977; Henriksen et al. 1997). The high densities measured here may be a result of the stage of evolution of the individual clumps.

Formaldehyde observations also provide important evidence for a large warm envelope. While the low-lying lines observed in this study are predominantly excited in the massive outer envelope, the derived temperature places constraints on the required heating source. In particular, envelope temperatures above $20 \mathrm{~K}$ are difficult to reconcile without either a warming source inside or an external heating source. All the sources observed in this study have two components, narrow and broad, associated with the formaldehyde measurements. However, this is not always the case for sub-millimeter bright regions (Mitchell et al. 2001; Tothill, private communication). It is possible that the broad component of the formaldehyde, often implying a much denser zone, is tracing an inner region within the envelope which is undergoing collapse or has become much more turbulent.

Not all heating is due to protostellar sources and it is therefore important to distinguish external heating (such as in a PDR) from internal heating. Protostellar sources should have central condensations which show up both in sub-millimeter dust continuum maps and maps of molecular lines with high critical densities (e.g., CS 7-6). Chemically, PDRs are best recognized by a high $[\mathrm{HCN}] /[\mathrm{HNC}]$ ratio and by high abundances of radicals such as $\mathrm{CN}$ and $\mathrm{C}_{2} \mathrm{H}$ and ions like $\mathrm{CO}^{+}$(see above discussion). 


\subsection{Line contamination}

One of the original motivations for this molecular line study was to determine the importance of line contamination within the broad SCUBA $850 \mu \mathrm{m}$ passband. While theoretical calculations (Appendix A) show that the influence of $\mathrm{CO}$ contamination can become exceedingly large in particular situations, it is also clear that typical conditions within molecular clouds are not so extreme. The observational evidence presented (Table 5) does show that the contamination, while typically less than $10 \%$, occasionally dominates the continuum flux. However, this only occurs in regions with warmer molecular gas temperatures and large velocity gradients which allow for enhanced integrated line strengths in $\mathrm{CO}$ and its isotopes. Such conditions occur most often at isolated shock fronts within the cloud, for example in the knots associated with protostellar jets. Contamination may also arise from lines of other molecules, especially around energetic sources. The best observed example for this is Orion KL, for which a forest of lines, primarily from $\mathrm{SO}$ and $\mathrm{SO}_{2}$, produce between $28 \%$ and $50 \%$ line contamination at $850 \mu \mathrm{m}$ (Serabyn \& Weisstein 1995; Groesbeck 1994). Consideration of Table 5 shows that in our sample the line contamination is never dominated by lines other than $\mathrm{CO}$; however, for the most energetic sources line emission from $\mathrm{HCN}, \mathrm{HNC}, \mathrm{CN}$, and methanol provides a significant fraction $(>40 \%)$ of the total line contamination.

Acknowledgements. We wish to thank Jes Jørgensen, Floris van der Tak, John Bally and Heather Scott for helpful comments during the creation of this manuscript. The careful review by the anonymous referee produced many improvements to the paper. DJ wishes to thank the Sterrewacht Leiden for its kind hospitality during the past three summers, at which time most of the data analysis was conducted. DJ also acknowledges the support of an NSERC grant and support from NOVA. This work was supported by the Netherlands Organization for Scientific Research (NWO) through grant 614-041-003 and a Spinoza grant.

\section{Appendix A: Contamination of sub-millimeter continuum by molecular lines}

Comparison of $850 \mu \mathrm{m}$ continuum against CO 3-2 measurements in the spiral arms of external disk galaxies has revealed that as much as $50 \%$ of the emission within the SCUBA bandpass is contamination due to this single line (Tilanus et al. 2000). This result is not surprising since the energy radiated in an optically thin $\mathrm{CO} j-i$ line by a column of gas with gas temperature $T_{\mathrm{g}}$ and number density $n$ is given by

$S_{j i}=\left(\frac{h v_{j i}}{4 \pi}\right) A_{j i} N(\mathrm{CO}) X_{\mathrm{j}}\left(T_{\mathrm{g}}, n\right) \quad \operatorname{erg~s}^{-1} \mathrm{~cm}^{-2} \mathrm{sr}^{-1}$,

where the column of $\mathrm{CO}$ is $N(\mathrm{CO})=x(\mathrm{CO}) N\left(\mathrm{H}_{2}\right)$, and the fractional population of the jth level is $X_{j}$. For the CO 3-2 line, $v_{32}=3.458 \times 10^{11} \mathrm{~s}^{-1}$ and $A_{32}=2.48 \times 10^{-6} \mathrm{~s}^{-1}$. Alternatively, for optically thin dust the energy radiated across a passband centered at $v_{\mathrm{d}}$ and with an effective width $\Delta v$ is

$S_{\mathrm{d}}=B_{v_{\mathrm{d}}}\left(T_{\mathrm{d}}\right) N_{\mathrm{H}_{2}} \mu m_{\mathrm{H}} \kappa_{v_{\mathrm{d}}} \Delta v \quad \operatorname{erg~s}^{-1} \mathrm{~cm}^{-2} \mathrm{sr}^{-1}$,
Table A.1. Fractional population of the 3rd CO energy level for different physical conditions.

\begin{tabular}{lccc}
\hline \hline & \multicolumn{3}{c}{ Density $\left(\mathrm{cm}^{-3}\right)$} \\
Temperature $(\mathrm{K})$ & $10^{3}$ & $10^{4}$ & $10^{5}$ \\
\hline 10 & $2.51 \mathrm{e}-3$ & $2.23 \mathrm{e}-2$ & $5.51 \mathrm{e}-2$ \\
20 & $1.23 \mathrm{e}-2$ & $9.02 \mathrm{e}-2$ & $1.68 \mathrm{e}-1$ \\
30 & $2.21 \mathrm{e}-2$ & $1.41 \mathrm{e}-1$ & $2.13 \mathrm{e}-1$ \\
50 & $3.66 \mathrm{e}-2$ & $1.95 \mathrm{e}-1$ & $2.24 \mathrm{e}-1$ \\
100 & $5.61 \mathrm{e}-2$ & $2.38 \mathrm{e}-1$ & $1.91 \mathrm{e}-1$ \\
500 & $8.44 \mathrm{e}-2$ & $2.53 \mathrm{e}-1$ & $1.13 \mathrm{e}-1$ \\
\hline
\end{tabular}

where $\kappa_{v_{\mathrm{d}}}$ is the emissivity of the dust at $\nu_{\mathrm{d}}$ and $\mu$ is the mean molecular weight of the gas. Since the dust radiation in molecular clouds is usually near the Rayleigh-Jeans limit, one can replace the Planck function $B_{v}\left(T_{\mathrm{d}}\right)$ with $2 k T_{\mathrm{d}} f_{v}\left(T_{\mathrm{d}}\right) / \lambda_{v}^{2}$, where $f_{v}\left(T_{\mathrm{d}}\right)$ accounts for the difference between the Planck law and the Rayleigh-Jeans limit at frequency $v$.

Thus, in the optically thin limit the ratio of the line to continuum is independent of the column of hydrogen, depending only on the temperature and density within the region, the abundance of $\mathrm{CO}$ in the gas phase, and the dust emissivity.

$R=\frac{1}{8 \pi}\left(\frac{h v_{j i}}{k T_{\mathrm{d}}}\right)\left(\frac{A_{j i}}{\Delta v}\right)\left(\frac{\lambda_{v_{\mathrm{d}}}^{2}}{\mu m_{\mathrm{H}} \kappa_{v_{\mathrm{d}}}}\right) f_{v_{\mathrm{d}}}\left(T_{\mathrm{d}}\right) x(\mathrm{CO}) X_{3}\left(T_{\mathrm{g}}, n\right)$. (A.3)

Computing the ratio of the $\mathrm{CO} 3-2$ line against the JCMT $850 \mu \mathrm{m}$ continuum band, where $\Delta v=3 \times 10^{10} \mathrm{~s}^{-1}$, yields

$$
\begin{aligned}
R_{850}= & 28\left(\frac{T_{\mathrm{d}}}{20 \mathrm{~K}}\right)^{-1}\left(\frac{\kappa_{850}}{0.02 \mathrm{~cm}^{2} / \mathrm{g}}\right)^{-1}\left(\frac{x(\mathrm{CO})}{1 \times 10^{-4}}\right) \\
& \times f_{850}\left(T_{\mathrm{d}}\right) X_{3}\left(T_{\mathrm{g}}, n\right) .
\end{aligned}
$$

The ratio depends strongly on the population of the upper $\mathrm{CO}$ energy level $X_{3}$ which is a function of both the gas temperature and the density. Tables A. 1 and A.2 show $X_{3}$ and $R_{850}$ for a range of internal gas conditions, assuming that the gas dust temperatures are equal. The values shown in Table A.1 only apply in the optically thin limit for the CO 3-2 transition $\left(N\left(\mathrm{H}_{2}\right)<10^{21} \mathrm{~cm}^{-3}\right)$. At higher columns, as are expected deep within molecular clouds, the continuum emission continues to increase linearly while the line emission is severely damped and the contamination is expected to be less influential. For extragalactic studies, where the spatial resolution does not permit the separation of thick and thin portions of the cloud, it is not surprising that the $\mathrm{CO}$ emission produces a significant contamination.

The degree to which the CO line contamination affects sub-millimeter continuum measurements at high optical depth within molecular clouds, as investigated in this paper, depends sensitively on the width of the CO 3-2 line and the strength of the extended line wings, and should be measured independently where possible. However, as long as the line is not significantly broadened, the $\mathrm{CO}$ contamination should be minor. Freeze-out of the $\mathrm{CO}$ onto dust grains decreases the importance of $\mathrm{CO}$ contamination for the densest and coldest regions. 
Table A.2. Ratio of CO line strength to $850 \mu \mathrm{m}$ continuum with SCUBA for different physical conditions, assuming $[\mathrm{CO}] /\left[\mathrm{H}_{2}\right]=10^{-4}$ and $\kappa_{850}=0.02 \mathrm{~cm}^{2} \mathrm{~g}^{-1}$.

\begin{tabular}{llll}
\hline \hline & Density $\left(\mathrm{cm}^{-3}\right)$ & & \\
Temperature $(\mathrm{K})$ & $10^{3}$ & $10^{4}$ & $10^{5}$ \\
\hline 10 & 0.4 & 3.3 & 8.2 \\
20 & 0.8 & 4.0 & 7.5 \\
30 & 0.6 & 3.6 & 5.4 \\
50 & 0.5 & 2.6 & 3.0 \\
100 & 0.4 & 1.5 & 1.2 \\
500 & 0.1 & 0.3 & 0.1 \\
\hline
\end{tabular}

\section{References}

Aso, Y., Tatematsu, K., Sekimoto, Y., et al. 2000, ApJS, 131, 465 Bachiller, R., \& Pérez-Gutiérrez, M. 1997, ApJ, 487, 93L

Bachiller, R., Liechti, S., Walmsley, C. M., \& Colomer, F. 1995, A\&A, 295, 51

Bacmann, A., Lefloch, B., Ceccarelli, C., et al. 2002, A\&A, 389, 6

Bally, J., \& Lada, C. J. 1983, ApJ, 265, 824

Bally, J., Langer, W. D., Stark, A. A., \& Wilson, R. W. 1987, ApJ, 313, L45

Bergin, E. A., Alves, J., Huard, T., \& Lada, C. J. 2002, ApJ, 570, L101 Blake, G. A., Sutton, E. C., Masson, C. R., \& Phillips, T. G. 1987, ApJ, 315, 621

Blake, G. A., van Dishoeck, E. F., Jansen, D. J., Groesbeck, T. D., \& Mundy, L. G. 1994, ApJ, 428, 680

Blake, G. A., Sandell, G., van Dishoeck, E. F., et al. 1995, ApJ, 441, 689

Boonman, A. M. S., Stark, R., van der Tak, F. F. S., et al. 2001, ApJ, $553,63 \mathrm{~L}$

Boonman, A. M. S., \& van Dishoeck, E. F. 2003, A\&A, 403, 1003

Boonman, A. M. S., Doty, S. D., van Dishoeck, E. F., et al. 2003, A\&A, 406, 937

Buckle, J. V., \& Fuller, G. A. 2002, A\&A, 381, 77

Butner, H. M., Evans, N. J. II, Harvey, P. M., et al. 1990, ApJ, 364, 164

Castets, A., \& Langer, W. D. 1995, A\&A, 294, 835

Charnley, S. B. 1997, ApJ, 481, 396

Charnley, S. B., Tielens, A. G. G. M., \& Millar, T. J. 1992, ApJ, 399, L71

Ceccarelli, C., Loinard, L., Castets, A., Tielens, A. G. G. M., \& Caux, E. 2000, A\&A, 357, L9

Chernin, L. M., Masson, C. R., \& Fuller, G. A. 1994, ApJ, 436, 741

Chini, R., Reipurth, B., Ward-Thompson, D., et al. 1997, ApJ, 474, L135

Collings, M. P., Dever, J. W., Fraser, H. J., \& McCoustra, M. R. S. 2003, ApJ, 583, 1058

Dartois, E., Schutte, W., Geballe, T. R., et al. 1999, A\&A, 342, L32

Doty, S. D., van Dishoeck, E. F., van der Tak, F. F. S., \& Boonman, A. M. S. 2002, A\&A, 389, 908

Flower, D. R. 2001, J. Phys. B, 34, 2731

Fraser, H. J., Collings, M. P., McCoustra, M. R. S., \& Williams, D. A. 2001, MNRAS, 327, 1165

Fuente, A., Martin-Pintado, J., Bachiller, R., Neri, R., \& Palla, F. 1998, A\&A, 334

Gibb, E. L., Whittet, D. C. B., Schutte, W. A., et al. 2000, ApJ, 536, 347

Green, S. 1991, ApJS, 76, 979

Green, S., \& Thaddeus, P. 1974, ApJ, 191, 653

Groesbeck, T. D. 1994, Ph.D. Thesis, Caltech
Harvey, P. M., Campbell, M. F., Hoffmann, W. F., Thronson, H. A., Jr., \& Gatley, I. 1979, ApJ, 229, 990

Hatchell, J., Thompson, M. A., Millar, T. J., \& MacDonald, G. H. 1998, A\&AS, 133, 29

Henriksen, R., André, P., \& Bontemps, S. 1997, A\&A, 323, 549

Helmich, F. P., \& van Dishoeck, E. F. 1997, A\&AS, 124, 205

Hillenbrand, L. A. 1997, AJ, 113, 1733

Hillenbrand, L. A., \& Hartmann, L. W. 1998, ApJ, 492, 540

Hogerheijde, M. R., Jansen, D. J., \& van Dishoeck, E. F. 1995, A\&A, 294, 792

Hurt, R. L., Barsony, M., \& Wootten, A. 1996, ApJ, 456, 686

Jansen, D. J., van Dishoeck, E. F., \& Black, J. H. 1994, A\&A, 282, 605

Jansen, D. J., Spaans, M., Hogerheijde, M. R., \& van Dishoeck, E. F. 1995, A\&A, 303, 541

Johnson, J. J., Gehrz, R. D., Jones, T. J., Hackwell, J. A., \& Grasdalen, G. L. 1990, AJ, 100, 518

Johnstone, D., \& Bally, J. 1999, ApJ, 510, L49

Johnstone, D., Fich, M., Mitchell, G. F., \& Moriarty-Schieven, G. 2001, ApJ, 559, 307

Jørgensen, J. K., Schöier, F. L., \& van Dishoeck, E. F. 2002, A\&A, 389, 908

Jørgensen, J. K., Schöier, F. L., \& van Dishoeck, E. F. 2003, in Chemistry as a Diagnostic of Star Formation, ed. C. L. Curry, \& M. Fich, in press

Keane, J. V., Boonman, A. M. S., Tielens, A. G. G. M., \& van Dishoeck, E. F. 2001a, A\&A, 379, 588

Keane, J. V., Tielens, A. G. G. M., Boogert, A. C. A., Schutte, W. A., \& Whittet, D. C. B. 2001b, A\&A, 376, 254

Lahuis, F., \& van Dishoeck, E. F. 2000, A\&A, 355, L699

Leen, T. M., \& Graff, M. M. 1988, ApJ, 325, 411

Lees, R., \& Haque, S. 1974, Canadian J. of Physics, 52, 2250

Li, D., Goldsmith, P. F., \& Menten, K. 2003, ApJ, 587, 262

Lis, D. C., Serabyn, E., Keene, J., et al. 1998, ApJ, 509, L299

MacDonald, G. H., Gibb, A. G., Habing, R. J., \& Millar, T. J. 1996, A\&AS, 119, 333

Mangum, J. G., \& Wootten, A. 1993, ApJS, 89, 123

Mangum, J. G., Wootten, A., \& Barsony, M. 1999, ApJ, 526, 845

Mezger, P. G., Zylka, R., \& Wink, J. E. 1990, A\&A, 228, 95

Mitchell, G., Johnstone, D., Moriarty-Schieven, G., Fich, M., \& Tothill, N. F. H. 2001, ApJ, 513, 139

Motte, F., André, P., Ward-Thompson, D., \& Bontemps, S. 2001, A\&A, 372, L41

Myers, P. C. 1999, in The Origin of Stars and Planetary Systems, ed. C. J. Lada, \& N. D. Kylafis (Kluwer Academic Publishers), 67

Reipurth, B., \& Aspin, C. 1997, AJ, 114, 2700

Reipurth, B., Bally, J., \& Devine, D. 1997, AJ, 114, 2708

Reipurth, B., Rodriguez, L. F., \& Chini, R. 1999, AJ, 118, 983

Rodgers, S. D., \& Charnley, S. B. 2001, ApJ, 546, 324

Schilke, P., Walmsley, C. M., Pineau Des Forêts, G., et al. 1992, A\&A, 256, 595

Schinke, R., Engel, V., Buck, U., Meyer, H., \& Diercksen, G. H. F. 1985, ApJ, 299, 939

Schöier, F. L., Jørgensen, J. K., van Dishoeck, E. F., \& Blake, G. A. 2002, A\&A, 390, 1001

Schöier, F. L., Jørgensen, J. K., van Dishoeck, E. F., \& Blake, G. A. 2003, in Chemistry as a Diagnostic of Star Formation, ed. C. L. Curry, \& M. Fich, in press

Schreyer, K., Henning, Th., van der Tak, F. F. S., Boonman, A. M. S., \& van Dishoeck, E. F. 2002, A\&A, 394, 561

Serabyn, E., \& Weisstein, E. W. 1995, ApJ, 451, 238

Shirley, Y. L., Evans, N. J. II, \& Rawlings, J. M. C. 2002, ApJ, 575, 337 
Shu, F. 1977, ApJ, 214, 488

Tielens, A. G. G. M., \& Charnley, S. B. 1997, Origins of Life and Evolution of the Biosphere, 27, 23

Tilanus, R. P. J., van der Werf, P. P., \& Israel, F. P. 2000, in Imaging at Radio through Submillimeter Wavelengths, ed. J. G. Mangum, \& S. J. E. Radford, ASP Conf. Ser., 217, 177

van der Tak, F. F. S., van Dishoeck, E. F., \& Caselli, P. 2000b, A\&A, 361,327

van der Tak, F. F. S., van Dishoeck, E. F., Evans, N. J. II, \& Blake, G. A. 2000a, ApJ, 537, 283

van der Tak, F. F. S., Boonman, A. M. S., Braakman, R., \& van Dishoeck, E. F. 2003, A\&A, 412, 133 van Dishoeck, E. F., \& Blake, G. A. 1998, ARA\&A, 36, 317

van Dishoeck, E. F., \& Helmich, F. P. 1996, A\&A, 315, L177

van Dishoeck, E. F., Jansen, D. J., \& Phillips, T. G. 1993, A\&A, 279, 541

van Dishoeck, E. F., Blake, G. A., Jansen, D. J., \& Groesbeck, T. D. 1995, ApJ, 447, 760

Wilson, T. L., \& Rood, R. 1994, ARA\&A, 32, 191

Wolstencroft, R. D., Scarrott, S. M., Warren-Smith, R. F., et al. 1986, MNRAS, 218, 1

Yu, K. C., Bally, J., \& Devine, D. 1997, ApJ, 485, L45

Yu, K. C., Billawala, Y., Smith, M. D., Bally, J., \& Butner, H. M. 2000, AJ, 120, 1974 
D. Johnstone et al.: Astrochemistry of sub-millimeter sources in Orion, Online Material p 1

\section{Online Material}


D. Johnstone et al.: Astrochemistry of sub-millimeter sources in Orion, Online Material $p 2$

Table 3. Observed spectra and Gaussian fit parameters.

\begin{tabular}{|c|c|c|c|c|c|c|}
\hline Source & Species & $\begin{array}{r}v \\
(\mathrm{GHz})\end{array}$ & $J$ & $\begin{array}{r}W \\
\left(\mathrm{~K} \mathrm{~km} \mathrm{~s}^{-1}\right)\end{array}$ & $\begin{array}{r}\Delta V \\
\left(\mathrm{~km} \mathrm{~s}^{-1}\right)\end{array}$ & $\begin{array}{r}T_{\mathrm{MB}} \\
(\mathrm{K})\end{array}$ \\
\hline \multirow[t]{29}{*}{ MMS6-OMC3 } & $\mathrm{CO}$ & 345.796 & $3-2$ & 93.00 & - & - \\
\hline & ${ }^{13} \mathrm{CO}$ & 330.588 & $3-2$ & 4.22 & 0.57 & 7.00 \\
\hline & & & & 40.70 & 2.24 & 17.1 \\
\hline & $\mathrm{C}^{17} \mathrm{O}$ & 337.061 & $3-2$ & 4.78 & 1.08 & 4.15 \\
\hline & $\mathrm{H}_{2} \mathrm{CO}$ & 218.222 & $3_{03}-2_{02}$ & 2.81 & 0.88 & 3.00 \\
\hline & & & & 2.52 & 2.40 & 0.99 \\
\hline & $\mathrm{H}_{2} \mathrm{CO}$ & 218.475 & $3_{22}-2_{21}$ & 0.53 & 1.73 & 0.29 \\
\hline & $\mathrm{H}_{2} \mathrm{CO}$ & 362.736 & $5_{05}-4_{04}$ & 1.80 & 0.91 & 1.85 \\
\hline & & & & 1.76 & 2.92 & 0.57 \\
\hline & $\mathrm{CH}_{3} \mathrm{OH}$ & 218.440 & $4_{2} E-3{ }_{1} E$ & 0.44 & 1.41 & 0.29 \\
\hline & $\mathrm{CH}_{3} \mathrm{OH}$ & 241.700 & $5_{0} \mathrm{E}-4_{0} \mathrm{E}$ & 0.18 & 0.82 & 0.21 \\
\hline & $\mathrm{CH}_{3} \mathrm{OH}$ & 241.767 & $5_{-1} \mathrm{E}-4_{-1} \mathrm{E}$ & 0.95 & 1.63 & 0.55 \\
\hline & $\mathrm{CH}_{3} \mathrm{OH}$ & 241.791 & $5_{0} \mathrm{~A}^{+}-4_{0} \mathrm{~A}^{+}$ & 1.27 & 1.65 & 0.72 \\
\hline & $\mathrm{CH}_{3} \mathrm{OH}$ & 241.904 & $5_{ \pm 2} \mathrm{E}-4_{ \pm 2} \mathrm{E}$ & 0.34 & 2.80 & 0.11 \\
\hline & $\mathrm{CH}_{3} \mathrm{OH}$ & 338.345 & $7_{-1} E-6_{-1} E$ & 0.84 & 1.56 & 0.50 \\
\hline & $\mathrm{CH}_{3} \mathrm{OH}$ & 338.404 & $7_{6} \mathrm{E}-6_{6} \mathrm{E}$ & 0.34 & 0.99 & 0.32 \\
\hline & & 338.409 & $7_{0} \mathrm{~A}^{+}-6_{0} \mathrm{~A}^{+}$ & 0.96 & 3.45 & 0.26 \\
\hline & $\mathrm{CH}_{3} \mathrm{OH}$ & 338.583 & $7_{3} \mathrm{E}-6_{3} \mathrm{E}$ & 0.51 & 4.81 & 0.10 \\
\hline & $\mathrm{CH}_{3} \mathrm{OH}$ & 338.615 & $7_{1} E-6_{1} E$ & 0.46 & 2.05 & 0.21 \\
\hline & $\mathrm{CH}_{3} \mathrm{OH}$ & 338.722 & $7_{ \pm 2} \mathrm{E}-6_{ \pm 2} \mathrm{E}$ & 0.45 & 1.69 & 0.25 \\
\hline & $\mathrm{CN}$ & 340.248 & $3-2$ & 2.95 & 1.41 & 1.97 \\
\hline & $\mathrm{HNC}$ & 362.630 & $4-3$ & 4.56 & 1.06 & 4.04 \\
\hline & $\mathrm{HCN}$ & 354.505 & $4-3$ & 9.65 & 3.81 & 2.38 \\
\hline & $\mathrm{H}^{13} \mathrm{CN}$ & 345.340 & $4-3$ & 0.49 & 2.05 & 0.23 \\
\hline & $\mathrm{CS}$ & 244.936 & $5-4$ & 2.31 & 0.93 & 2.34 \\
\hline & & & & 2.23 & 2.85 & 0.74 \\
\hline & $\mathrm{C}^{34} \mathrm{~S}$ & 241.016 & $5-4$ & 0.65 & 1.58 & 0.39 \\
\hline & $\mathrm{C}^{34} \mathrm{~S}$ & 337.396 & $7-6$ & 0.54 & 1.19 & 0.43 \\
\hline & SO & 346.528 & $8,9-7,8$ & 1.28 & 1.23 & 0.98 \\
\hline
\end{tabular}


D. Johnstone et al.: Astrochemistry of sub-millimeter sources in Orion, Online Material p 3

Table 3. continued.

\begin{tabular}{|c|c|c|c|c|c|c|}
\hline Source & Species & $\begin{array}{r}v \\
(\mathrm{GHz})\end{array}$ & $J$ & $\begin{array}{r}W \\
\left(\mathrm{~K} \mathrm{~km} \mathrm{~s}^{-1}\right) \\
\end{array}$ & $\begin{array}{r}\Delta V \\
\left(\mathrm{~km} \mathrm{~s}^{-1}\right)\end{array}$ & $\begin{array}{r}T_{\mathrm{MB}} \\
(\mathrm{K}) \\
\end{array}$ \\
\hline \multirow[t]{2}{*}{ MMS7-OMC3 } & $\mathrm{H}_{2} \mathrm{CO}$ & 218.222 & $3_{03}-2_{02}$ & 2.03 & 1.38 & 1.39 \\
\hline & $\mathrm{H}_{2} \mathrm{CO}$ & 218.475 & $3_{22}-2_{21}$ & 0.10 & 0.55 & 0.18 \\
\hline \multirow[t]{18}{*}{ MMS9-OMC3 } & $\mathrm{CO}$ & 345.796 & $3-2$ & 225.00 & - & - \\
\hline & ${ }^{13} \mathrm{CO}$ & 330.588 & $3-2$ & 59.10 & 2.40 & 23.2 \\
\hline & $\mathrm{C}^{17} \mathrm{O}$ & 337.061 & $3-2$ & 2.88 & 1.29 & 2.10 \\
\hline & $\mathrm{H}_{2} \mathrm{CO}$ & 218.222 & $3_{03}-2_{02}$ & 1.42 & 0.67 & 1.99 \\
\hline & & & & 1.65 & 2.11 & 0.73 \\
\hline & $\mathrm{H}_{2} \mathrm{CO}$ & 218.475 & $3_{22}-2_{21}$ & 0.28 & 0.74 & 0.36 \\
\hline & $\mathrm{H}_{2} \mathrm{CO}$ & 362.736 & $5_{05}-4_{04}$ & 0.94 & 1.12 & 0.78 \\
\hline & $\mathrm{CH}_{3} \mathrm{OH}$ & 218.440 & $4_{2} E-3{ }_{1} E$ & 0.23 & 0.98 & 0.22 \\
\hline & $\mathrm{CH}_{3} \mathrm{OH}$ & 241.700 & $5_{0} \mathrm{E}-4_{0} \mathrm{E}$ & 0.14 & 0.82 & 0.16 \\
\hline & $\mathrm{CH}_{3} \mathrm{OH}$ & 241.767 & $5_{-1} \mathrm{E}-4_{-1} \mathrm{E}$ & 0.51 & 1.21 & 0.40 \\
\hline & $\mathrm{CH}_{3} \mathrm{OH}$ & 241.791 & $5_{0} \mathrm{~A}^{+}-4_{0} \mathrm{~A}^{+}$ & 0.66 & 0.86 & 0.72 \\
\hline & $\mathrm{CH}_{3} \mathrm{OH}$ & 338.345 & $7_{-1} E-6_{-1} E$ & 0.30 & 0.55 & 0.50 \\
\hline & $\mathrm{CN}$ & 340.248 & $3-2$ & 4.65 & 1.78 & 2.46 \\
\hline & $\mathrm{HNC}$ & 362.630 & $4-3$ & 2.97 & 0.98 & 2.85 \\
\hline & $\mathrm{HCN}$ & 354.505 & $4-3$ & 1.05 & 0.82 & 1.21 \\
\hline & & & & 3.57 & 3.40 & 0.98 \\
\hline & $\mathrm{H}^{13} \mathrm{CN}$ & 345.340 & $4-3$ & 0.41 & 5.23 & 0.07 \\
\hline & $\mathrm{CS}$ & 244.936 & $5-4$ & 2.73 & 1.50 & 1.71 \\
\hline
\end{tabular}


D. Johnstone et al.: Astrochemistry of sub-millimeter sources in Orion, Online Material p 4

Table 3. continued.

\begin{tabular}{|c|c|c|c|c|c|c|}
\hline Source & Species & $\begin{array}{r}v \\
(\mathrm{GHz})\end{array}$ & $J$ & $\begin{array}{r}W \\
\left(\mathrm{~K} \mathrm{~km} \mathrm{~s}^{-1}\right)\end{array}$ & $\begin{array}{r}\Delta V \\
\left(\mathrm{~km} \mathrm{~s}^{-1}\right)\end{array}$ & $\begin{array}{r}T_{\mathrm{MB}} \\
(\mathrm{K})\end{array}$ \\
\hline \multirow[t]{47}{*}{ FIR4-OMC2 } & $\mathrm{CO}$ & 345.796 & $3-2$ & 442.00 & - & - \\
\hline & ${ }^{13} \mathrm{CO}$ & 330.588 & $3-2$ & 76.20 & 2.57 & 27.8 \\
\hline & & & & 24.70 & 12.5 & 1.85 \\
\hline & $\mathrm{C}^{17} \mathrm{O}$ & 337.061 & $3-2$ & 1.27 & 0.90 & 1.32 \\
\hline & & & & 2.70 & 1.65 & 1.54 \\
\hline & $\mathrm{H}_{2} \mathrm{CO}$ & 218.222 & $3_{03}-2_{02}$ & 9.82 & 1.49 & 6.21 \\
\hline & & & & 15.70 & 6.28 & 2.35 \\
\hline & $\mathrm{H}_{2} \mathrm{CO}$ & 218.475 & $3_{22}-2_{21}$ & 2.96 & 1.50 & 1.85 \\
\hline & & & & 4.49 & 5.81 & 0.73 \\
\hline & $\mathrm{H}_{2} \mathrm{CO}$ & 362.736 & $5_{05}-4_{04}$ & 5.24 & 1.20 & 4.09 \\
\hline & & & & 29.3 & 6.02 & 4.50 \\
\hline & $\mathrm{CH}_{3} \mathrm{OH}$ & 218.440 & $4_{2} \mathrm{E}-3_{1} \mathrm{E}$ & 3.43 & 0.98 & 3.29 \\
\hline & & & & 7.49 & 5.10 & 1.38 \\
\hline & $\mathrm{CH}_{3} \mathrm{OH}$ & 241.700 & $5_{0} \mathrm{E}-4_{0} \mathrm{E}$ & 2.39 & 1.36 & 1.65 \\
\hline & & & & 5.36 & 5.33 & 0.95 \\
\hline & $\mathrm{CH}_{3} \mathrm{OH}$ & 241.767 & $5_{-1} E-4_{-1} E$ & 4.73 & 1.29 & 3.44 \\
\hline & & & & 9.33 & 5.50 & 1.60 \\
\hline & $\mathrm{CH}_{3} \mathrm{OH}$ & 241.791 & $5_{0} \mathrm{~A}^{+}-4_{0} \mathrm{~A}^{+}$ & 5.93 & 1.36 & 4.11 \\
\hline & & & & 10.10 & 5.25 & 1.81 \\
\hline & $\mathrm{CH}_{3} \mathrm{OH}$ & 241.904 & $5_{ \pm 2} \mathrm{E}-4_{ \pm 2} \mathrm{E}$ & 2.35 & 1.54 & 1.43 \\
\hline & & & & 6.65 & 5.68 & 1.10 \\
\hline & $\mathrm{CH}_{3} \mathrm{OH}$ & 338.345 & $7_{-1} E-6_{-1} E$ & 5.86 & 1.34 & 4.12 \\
\hline & & & & 21.70 & 5.28 & 3.85 \\
\hline & $\mathrm{CH}_{3} \mathrm{OH}$ & 338.404 & $7_{6} \mathrm{E}-6_{6} \mathrm{E}$ & 5.31 & 1.21 & 4.14 \\
\hline & & & & 25.20 & 5.30 & 4.46 \\
\hline & $\mathrm{CH}_{3} \mathrm{OH}$ & 338.513 & $7_{4} \mathrm{~A}^{ \pm}-6_{4} \mathrm{~A}^{ \pm}$ & 1.58 & 0.57 & 2.59 \\
\hline & & & & 13.30 & 7.37 & 1.69 \\
\hline & $\mathrm{CH}_{3} \mathrm{OH}$ & 338.542 & $7_{3} \mathrm{~A}^{ \pm}-6_{4} \mathrm{~A}^{ \pm}$ & 5.37 & 2.29 & 2.20 \\
\hline & & & & 11.30 & 3.54 & 3.00 \\
\hline & $\mathrm{CH}_{3} \mathrm{OH}$ & 338.583 & $7_{3} \mathrm{E}-6_{3} \mathrm{E}$ & 1.44 & 3.03 & 0.45 \\
\hline & & & & 1.96 & 5.70 & 0.32 \\
\hline & $\mathrm{CH}_{3} \mathrm{OH}$ & 338.615 & $7_{1} \mathrm{E}-6_{1} \mathrm{E}$ & 2.24 & 1.26 & 1.67 \\
\hline & & & & 10.20 & 5.68 & 1.69 \\
\hline & $\mathrm{CH}_{3} \mathrm{OH}$ & 338.640 & $7_{2} \mathrm{~A}^{+}-6_{2} 0 \mathrm{~A}^{+}$ & 9.44 & 4.19 & 2.12 \\
\hline & $\mathrm{CH}_{3} \mathrm{OH}$ & 338.722 & $7_{ \pm 2} \mathrm{E}-6_{ \pm 2} \mathrm{E}$ & 11.60 & 2.70 & 4.03 \\
\hline & & & & 29.20 & 7.23 & 3.79 \\
\hline & $\mathrm{CN}$ & 340.248 & $3-2$ & 7.48 & 1.88 & 3.73 \\
\hline & & & & 15.50 & 8.68 & 1.67 \\
\hline & HNC & 362.630 & $4-3$ & 9.63 & 1.62 & 5.60 \\
\hline & & & & 11.50 & 6.83 & 1.58 \\
\hline & $\mathrm{HCN}$ & 354.505 & $4-3$ & 163.00 & 10.2 & 15.0 \\
\hline & $\mathrm{H}^{13} \mathrm{CN}$ & 345.340 & $4-3$ & 8.66 & 8.43 & 0.97 \\
\hline & $\mathrm{C}^{34} \mathrm{~S}$ & 241.016 & $5-4$ & 0.72 & 1.40 & 0.48 \\
\hline & & & & 1.31 & 5.18 & 0.24 \\
\hline & $\mathrm{C}^{34} \mathrm{~S}$ & 337.396 & $7-6$ & 2.64 & 7.39 & 0.34 \\
\hline & $\mathrm{SO}$ & 346.528 & $8,9-7,8$ & $<9.00$ & 6.00 & 0.97 \\
\hline & & & & 6.70 & 6.00 & 1.10 \\
\hline
\end{tabular}


D. Johnstone et al.: Astrochemistry of sub-millimeter sources in Orion, Online Material p 5

Table 3. continued.

\begin{tabular}{|c|c|c|c|c|c|c|}
\hline Source & Species & $\begin{array}{r}v \\
(\mathrm{GHz})\end{array}$ & $J$ & $\begin{array}{r}W \\
\left(\mathrm{~K} \mathrm{~km} \mathrm{~s}^{-1}\right)\end{array}$ & $\begin{array}{r}\Delta V \\
\left(\mathrm{~km} \mathrm{~s}^{-1}\right)\end{array}$ & $\begin{array}{r}T_{\mathrm{MB}} \\
(\mathrm{K})\end{array}$ \\
\hline \multirow[t]{34}{*}{ NGC 2071IR } & $\mathrm{CO}$ & 345.796 & $3-2$ & 770.00 & - & - \\
\hline & $\mathrm{C}^{17} \mathrm{O}$ & 337.061 & $3-2$ & 2.96 & 1.73 & 1.60 \\
\hline & & & & 4.25 & 7.31 & 0.55 \\
\hline & $\mathrm{H}_{2} \mathrm{CO}$ & 218.222 & $3_{03}-2_{02}$ & 12.00 & 2.21 & 5.10 \\
\hline & & & & 14.00 & 9.12 & 1.45 \\
\hline & $\mathrm{H}_{2} \mathrm{CO}$ & 218.475 & $3_{22}-2_{21}$ & 3.75 & 2.95 & 1.19 \\
\hline & & & & 3.67 & 16.3 & 0.21 \\
\hline & $\mathrm{H}_{2} \mathrm{CO}$ & 362.736 & $5_{05}-4_{04}$ & 10.90 & 2.07 & 4.93 \\
\hline & & & & 10.70 & 7.50 & 1.34 \\
\hline & $\mathrm{CH}_{3} \mathrm{OH}$ & 218.440 & $4_{2} E-3{ }_{1} E$ & 3.12 & 2.30 & 1.28 \\
\hline & $\mathrm{CH}_{3} \mathrm{OH}$ & 241.700 & $5_{0} \mathrm{E}-4_{0} \mathrm{E}$ & 2.39 & 1.36 & 1.65 \\
\hline & & & & 5.36 & 5.33 & 0.94 \\
\hline & $\mathrm{CH}_{3} \mathrm{OH}$ & 241.767 & $5_{-1} E-4_{-1} E$ & 6.51 & 2.38 & 2.56 \\
\hline & & & & 6.18 & 12.1 & 0.48 \\
\hline & $\mathrm{CH}_{3} \mathrm{OH}$ & 241.791 & $5_{0} \mathrm{~A}^{+}-4_{0} \mathrm{~A}^{+}$ & 4.93 & 1.80 & 2.58 \\
\hline & & & & 8.12 & 7.25 & 1.05 \\
\hline & $\mathrm{CH}_{3} \mathrm{OH}$ & 241.879 & $5_{1} \mathrm{E}-4_{1} \mathrm{E}$ & 1.45 & 1.88 & 0.73 \\
\hline & $\mathrm{CH}_{3} \mathrm{OH}$ & 241.888 & $5_{2} \mathrm{~A}^{+}-4_{2} \mathrm{~A}^{+}$ & 0.74 & 2.24 & 0.31 \\
\hline & $\mathrm{CH}_{3} \mathrm{OH}$ & 241.904 & $5_{ \pm 2} \mathrm{E}-4_{ \pm 2} \mathrm{E}$ & 1.09 & 1.52 & 0.67 \\
\hline & & & & 2.98 & 4.66 & 0.60 \\
\hline & $\mathrm{CN}$ & 340.248 & $3-2$ & 14.50 & 2.61 & 5.20 \\
\hline & & & & 14.50 & 8.63 & 1.58 \\
\hline & $\mathrm{HNC}$ & 362.630 & $4-3$ & 12.30 & 1.92 & 6.00 \\
\hline & & & & 10.70 & 6.44 & 1.56 \\
\hline & $\mathrm{HN}^{13} \mathrm{C}$ & 345.340 & $4-3$ & 1.30 & 3.89 & 0.31 \\
\hline & $\mathrm{HCN}$ & 354.505 & $4-3$ & 52.10 & 3.95 & 12.4 \\
\hline & & & & 31.20 & 14.3 & 2.05 \\
\hline & $\mathrm{H}^{13} \mathrm{CN}$ & 345.340 & $4-3$ & 5.51 & 10.1 & 0.51 \\
\hline & $\mathrm{CS}$ & 244.936 & $5-4$ & 10.30 & 1.87 & 5.17 \\
\hline & & & & 25.50 & 6.29 & 3.81 \\
\hline & $C^{34} S$ & 241.016 & $5-4$ & 0.59 & 0.85 & 0.66 \\
\hline & & & & 3.86 & 3.45 & 1.05 \\
\hline & $\mathrm{C}^{34} \mathrm{~S}$ & 337.396 & $7-6$ & 0.53 & 3.70 & 0.13 \\
\hline & & & & 3.57 & 3.74 & 0.89 \\
\hline
\end{tabular}


D. Johnstone et al.: Astrochemistry of sub-millimeter sources in Orion, Online Material p 6

Table 3. continued.

\begin{tabular}{|c|c|c|c|c|c|c|}
\hline Source & Species & $\begin{array}{r}v \\
(\mathrm{GHz})\end{array}$ & $J$ & $\begin{array}{r}W \\
\left(\mathrm{~K} \mathrm{~km} \mathrm{~s}^{-1}\right)\end{array}$ & $\begin{array}{r}\Delta V \\
\left(\mathrm{~km} \mathrm{~s}^{-1}\right)\end{array}$ & $\begin{array}{r}T_{\mathrm{MB}} \\
(\mathrm{K})\end{array}$ \\
\hline \multirow[t]{23}{*}{ PDR1-OMC1 } & $\mathrm{CO}$ & 345.796 & $3-2$ & 535.00 & - & - \\
\hline & ${ }^{13} \mathrm{CO}$ & 330.588 & $3-2$ & 129.00 & 1.39 & 87.0 \\
\hline & & & & 90.10 & 1.28 & 66.0 \\
\hline & $\mathrm{C}^{17} \mathrm{O}$ & 337.061 & $3-2$ & 10.20 & 1.38 & 6.94 \\
\hline & $\mathrm{H}_{2} \mathrm{CO}$ & 218.222 & $3_{03}-2_{02}$ & 0.38 & 0.49 & 0.72 \\
\hline & & & & 5.11 & 1.88 & 2.55 \\
\hline & $\mathrm{H}_{2} \mathrm{CO}$ & 218.475 & $3_{22}-2_{21}$ & 0.23 & 0.49 & 0.43 \\
\hline & & & & 1.76 & 1.32 & 1.25 \\
\hline & $\mathrm{H}_{2} \mathrm{CO}$ & 362.736 & $5_{05}-4_{04}$ & 3.80 & 1.56 & 2.29 \\
\hline & $\mathrm{CH}_{3} \mathrm{OH}$ & 241.700 & $5_{0} \mathrm{E}-4_{0} \mathrm{E}$ & 0.33 & 1.83 & 0.17 \\
\hline & $\mathrm{CH}_{3} \mathrm{OH}$ & 241.767 & $5{ }_{-1} \mathrm{E}-4_{-1} \mathrm{E}$ & 0.89 & 1.87 & 0.45 \\
\hline & $\mathrm{CH}_{3} \mathrm{OH}$ & 241.791 & $5_{0} \mathrm{~A}^{+}-4_{0} \mathrm{~A}^{+}$ & 1.20 & 1.83 & 0.61 \\
\hline & $\mathrm{CH}_{3} \mathrm{OH}$ & 241.904 & $5_{ \pm 2} \mathrm{E}-4_{ \pm 2} \mathrm{E}$ & 0.35 & 1.71 & 0.19 \\
\hline & $\mathrm{CH}_{3} \mathrm{OH}$ & 338.345 & $7_{-1} E-6_{-1} E$ & 1.14 & 1.43 & 0.75 \\
\hline & $\mathrm{CH}_{3} \mathrm{OH}$ & 338.404 & $7_{6} E-6_{6} E$ & 1.85 & 1.86 & 0.94 \\
\hline & $\mathrm{CH}_{3} \mathrm{OH}$ & 338.722 & $7_{ \pm 2} \mathrm{E}-6_{ \pm 2} \mathrm{E}$ & 0.74 & 2.03 & 0.34 \\
\hline & $\mathrm{CN}$ & 340.248 & $3-2$ & 23.80 & 2.18 & 10.3 \\
\hline & $\mathrm{HNC}$ & 362.630 & $4-3$ & 3.83 & 1.57 & 2.29 \\
\hline & $\mathrm{HCN}$ & 354.505 & $4-3$ & 41.70 & 2.39 & 16.4 \\
\hline & $\mathrm{H}^{13} \mathrm{CN}$ & 345.340 & $4-3$ & 2.11 & 1.47 & 1.35 \\
\hline & $\mathrm{C}^{34} \mathrm{~S}$ & 241.016 & $5-4$ & 7.32 & 1.65 & 4.16 \\
\hline & $\mathrm{C}^{34} \mathrm{~S}$ & 337.396 & 7-6 & 5.74 & 1.28 & 4.21 \\
\hline & $\mathrm{SO}$ & 346.528 & $8,9-7,8$ & 4.82 & 1.64 & 2.77 \\
\hline \multirow[t]{10}{*}{ SK1-OMC3 } & $\mathrm{CO}$ & 345.796 & $3-2$ & 404.00 & - & - \\
\hline & ${ }^{13} \mathrm{CO}$ & 330.588 & $3-2$ & 5.33 & 1.12 & 4.49 \\
\hline & & & & 69.50 & 2.49 & 26.2 \\
\hline & $\mathrm{C}^{17} \mathrm{O}$ & 337.061 & $3-2$ & 3.31 & 1.60 & 1.94 \\
\hline & $\mathrm{H}_{2} \mathrm{CO}$ & 218.222 & $3_{03}-2_{02}$ & 0.57 & 1.45 & 0.37 \\
\hline & $\mathrm{H}_{2} \mathrm{CO}$ & 218.475 & $3_{22}-2_{21}$ & $<0.20$ & 0.49 & 0.37 \\
\hline & $\mathrm{CN}$ & 340.248 & $3-2$ & 0.94 & 3.49 & 0.25 \\
\hline & HNC & 362.630 & $4-3$ & 0.23 & 1.25 & 0.17 \\
\hline & $\mathrm{HCN}$ & 354.505 & $4-3$ & 0.43 & 1.24 & 0.32 \\
\hline & & & & 1.74 & 14.6 & 0.11 \\
\hline
\end{tabular}

